\title{
Forecasting with importance-sampling and path-integrals: Applications to COVID-19
}

\author{
Lester Ingber ${ }^{1}$
}

\begin{abstract}
:
Background: Forecasting nonlinear stochastic systems most often is quite difficult, without giving in to temptations to simply simplify models for the sake of permitting simple computations. Objective: Here, two basic algorithms, Adaptive Simulated Annealing (ASA) and path-integral codes PATHINT/PATHTREE (and their quantum generalizations qPATHINT/qPATHTREE) are offered to detail such systems. Method: ASA and PATHINT/PATHTREE have been effective to forecast properties in three disparate disciplines in neuroscience, financial markets, and combat analysis. Applications are described for COVID-19. Results: Results of detailed calculations have led to new results and insights not previously obtained. Conclusion: These 3 applications give strong support to a quite generic application of these tools to stochastic nonlinear systems.
\end{abstract}

Key words: path integral; importance sampling; neuroscience; financial markets; combat analysis; COVID-19

\section{Introduction}

This paper offers application of two basic algorithms to generic issues in forecasting.

(a) Adaptive Simulated Annealing (ASA) is an importance-sampling optimization code for nonlinear, nonequilibrium, non-stationary, multivariate systems.

(b) PATHINT is a numerical path-integral PATHINT code used for propagation of nonlinear probability distributions.

These codes were developed by the author and applied across multiple disciplines.

There is not "one size fits all" in forecasting. This is demonstrated for the three selected systems. It is reasonable to expect that this approach can be applied to future projects such as forecasting the spread of COVID-19.

\subsection{Technical considerations}

If there is not time to process large data sets, then the data can be randomly sampled, e.g., as described in previous papers (Ingber, 2020a).

If the required forecast is longer than the conditional distribution permits, PATHINT/PATHTREE can be used to propagate the distribution.

The dataset should be broken into independent Training and Testing subsets, to test the Trained distribution. If this is not possible, e.g., because of data or time limitations, at the least experts can be used to judge if the model is ready for real-time applications, e.g., the Delphi method (Okoli \& Pawlowski, 2004).

\footnotetext{
${ }^{1}$ Corresponding author: Lester Ingber

email: lester@ingber.com or ingber@caa.caltech.edu

Additional information may be obtained at https://www.ingber.com.
} 


\subsection{Outline}

Most major sections of this paper paraphrase other papers by this author.

Section 2 describes the ASA algorithm. If an algorithm is to be used across a large class of problems, then it must be tunable to different classes. Over the $30+$ years of ASA development, the author has worked with many volunteers who have contributed valuable ideas, modifications and corrections to the ASA code. This has resulted in over 150 ASA OPTIONS that can be used for additional across many classes of problems.

Section 3 describes the path integral algorithm and its mathematical equivalents, a large class of stochastic differential equations and a large class of partial differential equations. The advantages of the path integral algorithm are:

(a) intuitive description in terms of classical forces, inertia, momentum, etc., leading to new indicators.

(b) delivering a cost function derived from a Lagrangian, or its Action (Lagrangian x dt). Sometimes constraints need to be added as Lagrange multipliers, as was required for normalization requirements in financial risk projects (Ingber, 2010a).

Section 4 discusses applications to Statistical Mechanics of Neocortical Interactions (SMNI).

Section 5 discusses applications to Statistical Mechanics of Financial Markets (SMFM).

Section 6 discusses applications to Statistical Mechanics of Combat (SMC).

Section 7 describes possible applications to forecasting the spread of COVID-19.

Section 8 is the Conclusion.

\subsection{Model of models (MOM)}

It is likely that this work competes with many neural-network (NN) approaches to forecasting. The author has participated in NN research, applying ASA to optimize weights, and is aware of its strengths (Atiya et al., 2003).

Neural networks and their applications to Deep Learning (DL) have demonstrated great advances since a couple of decades ago when they fell out of favor because of risks of not adapting to changes of contexts of markets. This fear is still present and well-founded. The author has proposed an approach of Model of Models (MOM) to address this problem (Ingber, 2019).

Forecasting often requires audit trails back to basic assumptions, as a modeling approach offers.

The success of DL has overshadowed these concerns. However, that should not diminish the importance of these concerns, especially if such systems are placed in positions to affect lives and human concerns. Humans ultimately must be responsible for structures they build.

Model of Models (MOM) is an approach to dealing with these concerns. For thousands of years humans have developed models of reality across many disciplines, e.g., Physics, Biology, Mathematics, Economics, etc.

DL could process data for a given system, described in terms a collection of models, to determine a superior model of models (MOM). Eventually, large DL (quantum) machines could possess a database of hundreds or thousands of models across many disciplines, and directly find the best (hybrid) MOM for a given system.

ASA can be used in this expanded context of MOM, as a first step in a new system to which MOM is to be applied. This step would develop a range of parameters useful for DL, as DL by itself may get stuck in non-ideal local minima of the importance-sampled space. 
After a reasonable range of models is found, then DL permits much more efficient and accurate development of MOM for a given discipline/system.

\section{Adaptive Simulated Annealing (ASA)}

A paper discusses ASA in more detail (Ingber, 2012a). 


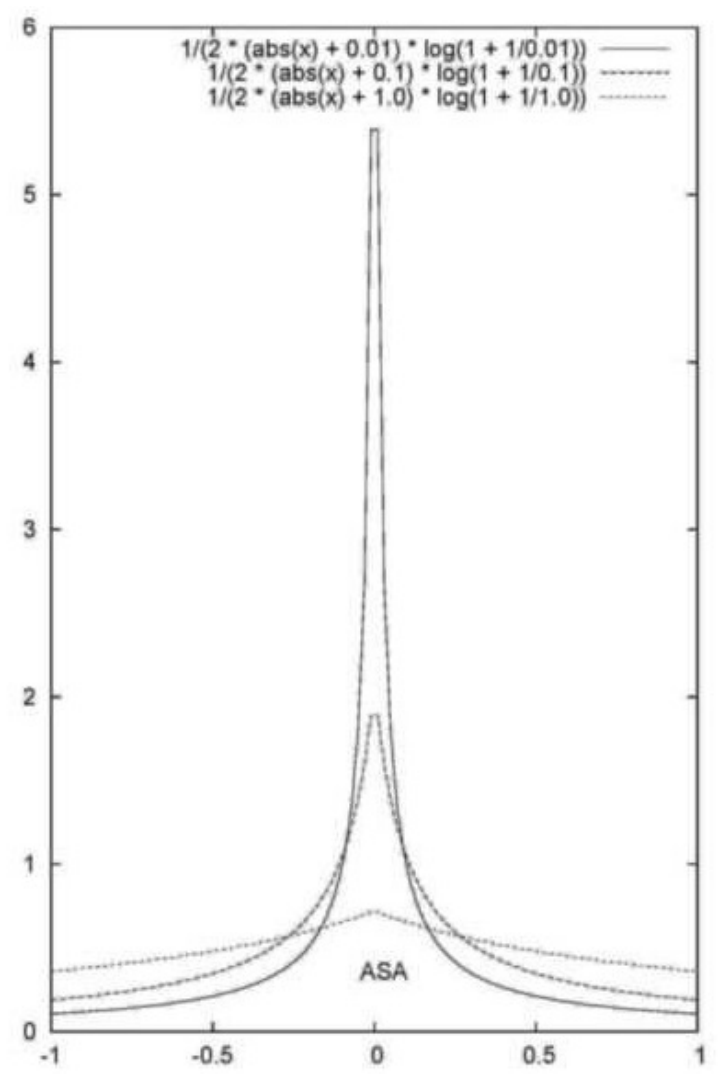

Fig. 1. The ASA distribution is given at various "temperatures".

Simulated annealing (SA) presents an optimization technique that: (a) processes cost functions with quite arbitrary degrees of nonlinearities, discontinuities, and stochasticity; (b) processes quite arbitrary boundary conditions and constraints; (c) can be implemented quite easily with a degree of coding minimal relative to other nonlinear optimization algorithms; (d) statistically guarantees finding an optimal solution.

Adaptive Simulated Annealing (ASA) is a C-language code that finds the best global fit of a nonlinear cost-function over a D-dimensional space. The basic algorithm was originally published as Very Fast Simulated Reannealing (VFSR) in 1989 (Ingber, 1989a), after two years of application on combat simulations for the US Navy and Army. The code (Ingber, 1993a) can be used at no charge and downloaded from http://www.ingber.com/\#ASA with mirrors at:

http://alumni.caltech.edu/ ingber http://asa-caltech.sourceforge.net

ASA currently has 152 OPTIONS to provide robust tuning over many classes of nonlinear stochastic systems. These OPTIONS help ASA to be used robustly across many classes of systems.

The "QUENCHing" OPTIONS are among the most important for controlling Adaptive Simulated Annealing. Fuzzy ASA algorithms in particular offer new ways of controlling how these QUENCHing OPTIONS may be applied across many classes of problems (Oliveira et al., 2012 ).

\subsection{Theoretical foundations of adaptive simulated annealing (ASA)}

The unique aspect of simulated annealing (SA) is its property of (weak) ergodicity, to statistically sample a parameter space. Note that for very large systems, ergodicity is not a rigorous concept when faced with the real task of its computation (Ma, 1985). "Ergodic" is used in a very weak 
sense, as it is not proposed, theoretically or practically, that all states of the system are actually to be visited.

\subsection{1 "Standard" simulated annealing (SA)}

The Metropolis Monte Carlo integration algorithm (Metropolis et al., 1953) was generalized by the Kirkpatrick algorithm to include a temperature schedule for efficient searching (Kirkpatrick et al., 1983), usually referred to as Boltzmann annealing (BA). A sufficiency proof was then shown to put an lower bound on that schedule as $1 / \log (t)$, where $t$ is an artificial time measure of the annealing schedule (Geman \& Geman, 1984). Independent credit usually goes to several other authors for independently developing the algorithm that is now recognized as simulated annealing (Cerny, 1982; Pincus, 1970).

\subsubsection{Boltzmann annealing (BA)}

Credit for the first simulated annealing is generally recognized as a Monte Carlo importancesampling technique for doing large-dimensional path integrals arising in statistical physics problems (Metropolis et al., 1953). This method was generalized to fitting non-convex cost-functions arising in a variety of problems, e.g., finding the optimal wiring for a densely wired computer chip (Kirkpatrick et al., 1983). The choices of probability distributions described are specified as Boltzmann annealing (BA) (Szu \& Hartley, 1987).

The method of simulated annealing consists of three algorithms.

1. $g(x)$ : Probability density of state-space of $D$ parameters $x=\left\{x^{i} ; i=1, D\right\}$.

2. $h(\Delta E)$ : Probability for acceptance of new cost-function given the just previous value.

3. $T(k)$ : schedule of "annealing" the "temperature" $T$ in annealing-time steps $k$, changing the volatility of one or both of the two previous probability densities.

The acceptance probability is based on the chances of obtaining a new state with "energy" $E_{k+1}$ relative to a previous state with "energy" $E_{k}$,

$$
\begin{aligned}
h(\Delta E)= & \frac{\exp \left(-E_{k+1} / T\right)}{\exp \left(-E_{k+1} / T\right)+\exp \left(-E_{k} / T\right)} \\
= & \frac{1}{1+\exp (\Delta E / T)} \\
& \approx \exp (-\Delta E / T)
\end{aligned}
$$

where $\Delta E$ represents the "energy" difference between the present and previous values of the energies specific to the problem, i.e., $\Delta E=E_{k+1}-E_{k}$. This is the Boltzmann distribution contributing to the statistical mechanical partition function of the system (Binder \& Stauffer, 1985).

This can be described as a set of states $x$, each with energy $e(x)$; a set of probability distributions $p(x)$; and the energy distribution per state $d(e(x))$, giving an aggregate energy $E$,

$$
\sum_{x} p(x) d(e(x))=E
$$

The principle of maximizing the entropy, $S$, 


$$
S=-\sum_{x} p(x) \ln [p(x) / p(\bar{x})]
$$

where $\bar{x}$ represents a reference state, using Lagrange multipliers (Mathews \& Walker, 1970) to constrain the energy to average value $T$ leads to the Gibbs distribution $G(x)$,

$$
G(x)=\frac{1}{Z} \exp (-H(x) / T)
$$

in terms of the normalizing partition function $Z$, and the Hamiltonian $H$ operator as the "energy" function,

$$
Z=\sum_{x} \exp (-H(x) / T)
$$

For such distributions of states and acceptance probabilities defined by functions such as $h(\Delta E)$, the equilibrium principle of detailed balance holds, wherein the distributions of states before, $G\left(x_{k}\right)$, and after, $G\left(x_{k+1}\right)$, applying the acceptance criteria, $h(\Delta E)=h\left(E_{k+1}-E_{k}\right)$ are the same:

$$
G\left(x_{k}\right) h(\Delta E(x))=G\left(x_{k+1}\right)
$$

This is sufficient to establish that all states of the system can be sampled, in theory. Since the annealing schedule interrupts equilibrium every time the temperature is changed, this must be done carefully and gradually.

An important aspect of the SA algorithm is to select ranges of parameters to be searched. In practice, computation of continuous systems requires some discretization. There are additional constraints required of generating and cost functions with integral values. Many users apply techniques to narrow the range as the search progresses. For example, one could choose an algorithm for $g$,

$$
g(\Delta x)=(2 \pi T)^{-D / 2} \exp \left[-\Delta x^{2} /(2 T)\right]
$$

where $\Delta x=x-x_{0}$ is the deviation of $x$ from $x_{0}$ proportional to a "momentum" variable, and where $T$ is a measure of the fluctuations of the Boltzmann distribution $g$ in the $D$-dimensional $x$-space. Given $g(\Delta x)$, it has been proven (Geman \& Geman, 1984) that it suffices to obtain a global minimum of $E(x)$ if $T$ is selected to be not faster than

$$
T(k)=\frac{T_{0}}{\ln k}
$$

with $T_{0}$ "large enough."

A heuristic demonstration shows that this choice of $T$ can suffice to give a global minimum of $E(x)$ (Szu \& Hartley, 1987). To statistically assure that any point in $x$-space can be sampled in annealing-time (IOT), it suffices to prove that the products of probabilities of not generating a state $x$ IOT for all annealing-times successive to $k_{0}$ yield zero,

$$
\prod_{k=k_{0}}^{\infty}\left(1-g_{k}\right)=0
$$

This is equivalent to

$$
\sum_{k=k_{0}}^{\infty} g_{k}=\infty
$$


The problem then reduces to finding $T(k)$ to satisfy this equation.

For BA, if $T(k)$ is selected to be the Boltzmann criteria above, then the generating distribution $g$ above gives

$$
\sum_{k=k_{0}}^{\infty} g_{k} \geq \sum_{k=k_{0}}^{\infty} \exp (-\ln k)=\sum_{k=k_{0}}^{\infty} 1 / k=\infty
$$

Although there are sound physical principles underlying the choices of the Boltzmann criteria above (Metropolis et al., 1953), this method of finding the global minimum in $x$-space is not limited to physics examples requiring This methodology can be extended to any problem for which a probability density $h(\Delta x)$ exists (Kirkpatrick et al., 1983).

\subsubsection{Fast annealing (FA)}

There are many variants and improvements made on the "standard" Boltzmann algorithm. Many users stop without going into detail about other algorithms (vanLaarhoven \& Aarts, 1987). For example, the Cauchy distribution has some advantages over the Boltzmann form (Szu \& Hartley, 1987). The Cauchy distribution,

$$
g(\Delta x)=\frac{T}{\left(\Delta x^{2}+T^{2}\right)^{(D+1) / 2}}
$$

has a "fatter" tail than the Gaussian form of the Boltzmann distribution, permitting wider access to local minima in the search for the global minimum.

The similar corresponding heuristic demonstration, that the Cauchy $g(\Delta x)$ statistically finds a global minimum, is obtained if the Boltzmann $T$ is replaced by

$$
T(k)=\frac{T_{0}}{k}
$$

Then

$$
\sum_{k_{0}}^{\infty} g_{k} \approx \frac{T_{0}}{\Delta x^{D+1}} \sum_{k_{0}}^{\infty} \frac{1}{k}=\infty
$$

This "normalization" of $g$ introduces the annealing-time index $k$, giving insights into how to construct other annealing distributions. The method of FA has an annealing schedule exponentially faster than the method of BA. This method has been tested in a variety of problems (Szu \& Hartley, 1987).

\subsubsection{Adaptive simulated annealing (ASA)}

Many problems are described in a $D$-dimensional parameter-space. Different parameters have different finite ranges, some fixed by physical considerations and different annealing-time-dependent sensitivities, e.g., as measured by the derivatives of the cost-function at local minima. Since $\mathrm{BA}$ and FA have distributions that sample infinite ranges, there is no provision for differences in each parameter-dimension; different sensitivities may require different annealing schedules. This prompted development of a probability distribution to accommodate these desired features (Ingber, 1989a), leading to a variant of SA that in fact justifies an exponential temperature annealing schedule. Several such considerations gave rise to Adaptive Simulated Annealing (ASA). The source code is publicly available at no charge without any registration (Ingber, 1993a). 
In ASA, parameter $\alpha_{k}^{i}$ in dimension $i$ generated at annealing-time $k$ with the range

$$
\alpha_{k}^{i} \in\left[A_{i}, B_{i}\right]
$$

is calculated using random variable $y^{i}$,

$$
\begin{gathered}
\alpha_{k+1}^{i}=\alpha_{k}^{i}+y^{i}\left(B_{i}-A_{i}\right) \\
y^{i} \in[-1,1]
\end{gathered}
$$

The generating function is defined

$$
g_{T}(y)=\prod_{i=1}^{D} \frac{1}{2\left(\left|y^{i}\right|+T_{i}\right) \ln \left(1+1 / T_{i}\right)} \equiv \prod_{i=1}^{D} g_{T}^{i}\left(y^{i}\right)
$$

Its cumulative probability distribution is

$$
\begin{gathered}
G_{T}(y)=\int_{-1}^{y^{1}} \ldots \int_{-1}^{y^{D}} d y^{\prime 1} \ldots d y^{\prime D} g_{T}\left(y^{\prime}\right) \equiv \prod_{i=1}^{D} G_{T}^{i}\left(y^{i}\right) \\
G_{T}^{i}\left(y^{i}\right)=\frac{1}{2}+\frac{\operatorname{sgn}\left(y^{i}\right)}{2} \frac{\ln \left(1+\left|y^{i}\right| / T_{i}\right)}{\ln \left(1+1 / T_{i}\right)}
\end{gathered}
$$

$y^{i}$ is generated from a $u^{i}$ from the uniform distribution

$$
\begin{gathered}
u^{i}[0,1] \\
y^{i}=\operatorname{sgn}\left(u^{i}-\frac{1}{2}\right) T_{i}\left[\left(1+1 / T_{i}\right)^{\left|2 u^{i}-1\right|}-1\right]
\end{gathered}
$$

For annealing schedule for $T_{i}$

$$
T_{i}(k)=T_{0 i} \exp \left(-c_{i} k^{1 / D}\right)
$$

a global minima statistically can be obtained, as seen by

$$
\sum_{k_{0}}^{\infty} g_{k} \approx \sum_{k_{0}}^{\infty}\left[\prod_{i=1}^{D} \frac{1}{2\left|y^{i}\right| c_{i}}\right] \frac{1}{k}=\infty
$$

Control over $c_{i}$ is obtained from

$$
\begin{gathered}
T_{f i}=T_{0 i} \exp \left(-m_{i}\right) \text { when } k_{f}=\exp n_{i} \\
c_{i}=m_{i} \exp \left(-n_{i} / D\right)
\end{gathered}
$$

where $m_{i}$ and $n_{i}$ are "free" parameters helping to tune ASA.

The same type of annealing schedule for the acceptance function $h$ can be used for the generating function $g$, but with the number of acceptance points instead of the number of generated points to determine the $k$ for the acceptance temperature.

New parameters $\alpha_{k+1}^{i}$ are generated from old parameters $\alpha_{k}^{i}$ from 


$$
\alpha_{k+1}^{i}=\alpha_{k}^{i}+y^{i}\left(B_{i}-A_{i}\right)
$$

constrained by

$$
\alpha_{k+1}^{i} \in\left[A_{i}, B_{i}\right]
$$

$y^{i}$ s are repeatedly generated until a set of $D$ are obtained satisfying these constraints.

\subsubsection{Quenching}

A strong adaptive feature of ASA is quenching, by noting that the temperature schedule above can be redefined as

$$
\begin{gathered}
T_{i}\left(k_{i}\right)=T_{0 i} \exp \left(-c_{i} k_{i}^{Q_{i} / D}\right) \\
c_{i}=m_{i} \exp \left(-n_{i} Q_{i} / D\right)
\end{gathered}
$$

in terms of "quenching factor" $Q_{i}$. The above proof of ergodic sampling fails if $Q_{i}>1$ as

$$
\sum_{k} \prod^{D} 1 / k^{Q_{i} / D}=\sum_{k} 1 / k^{Q_{i}}<\infty
$$

This shows how the "curse of dimensionality" arises, but gives a possible way of living with this curse. In ASA, the influence of large dimensions is focused on the exponential of the power of $k$ being $1 / D$, as the annealing required to properly sample the space becomes prohibitively slow. If resources cannot be committed to properly sample the space ergodically, then for some systems the next best procedure is to turn on quenching, whereby $Q_{i}$ can is on the order of the size of number of dimensions.

The scale of the power of the $1 / D$ temperature schedule used for the acceptance function can similarly be used. This does not affect the annealing proof of ASA, and this may be used without damaging the (weak) ergodicity property.

Many users take advantage of the ease of coding SA and its ability to handle quite complex cost functions and constraints. However, the long time of execution of standard Boltzmann-type SA too many times drives projects to a temperature schedule too fast to satisfy the sufficiency conditions required to establish a true (weak) ergodic search. Note that a logarithmic temperature schedule is consistent with the Boltzmann algorithm, e.g., the temperature schedule is taken to be

$$
T_{k}=T_{0} \frac{\ln k_{0}}{\ln k}
$$

where $T$ is the "temperature," $k$ is the "time" index of annealing, and $k_{0}$ is some starting index. For large $k$ this is

$$
\begin{gathered}
\Delta T=-T_{0} \frac{\ln k_{0} \Delta k}{k(\ln k)^{2}}, k \gg 1 \\
T_{k+1}=T_{k}-T_{0} \frac{\ln k_{0}}{k(\ln k)^{2}}
\end{gathered}
$$

Some researchers using the BA instead use an exponential schedule, e.g., 


$$
\begin{gathered}
T_{k+1}=c T_{k}, 0<c<1 \\
\frac{\Delta T}{T_{k}}=(c-1) \Delta k, k \gg 1 \\
T_{k}=T_{0} \exp ((c-1) k)
\end{gathered}
$$

with expediency the only reason given, without addressing or admitting the clear misuse of the claim to use SA to help solve these problems/systems? Adaptive simulated annealing (ASA) (Ingber, 1989a, 1993a), in fact does justify an exponential annealing schedule, but only if its ASA distribution is used for the generating function.

Often researchers know quite a bit about their system, the convenience of their chosen SA algorithm, and their need for global search over local optima. This can make a strong case for the use of simulated quenching (SQ). It must be stated that while SA's true strength lies in its ability to statistically deliver a true global optimum, there are no theoretical reasons for assuming it can be more efficient than any other algorithm that also can find the same global optimum. E.g., Newton's algorithm is often the best code for parabolic cost functions.

\section{PATHINT/PATHTREE and qPATHINT/qPATHTREE}

A paper discusses qPATHINT/qPATHTREE in more detail (Ingber, 2018a). 


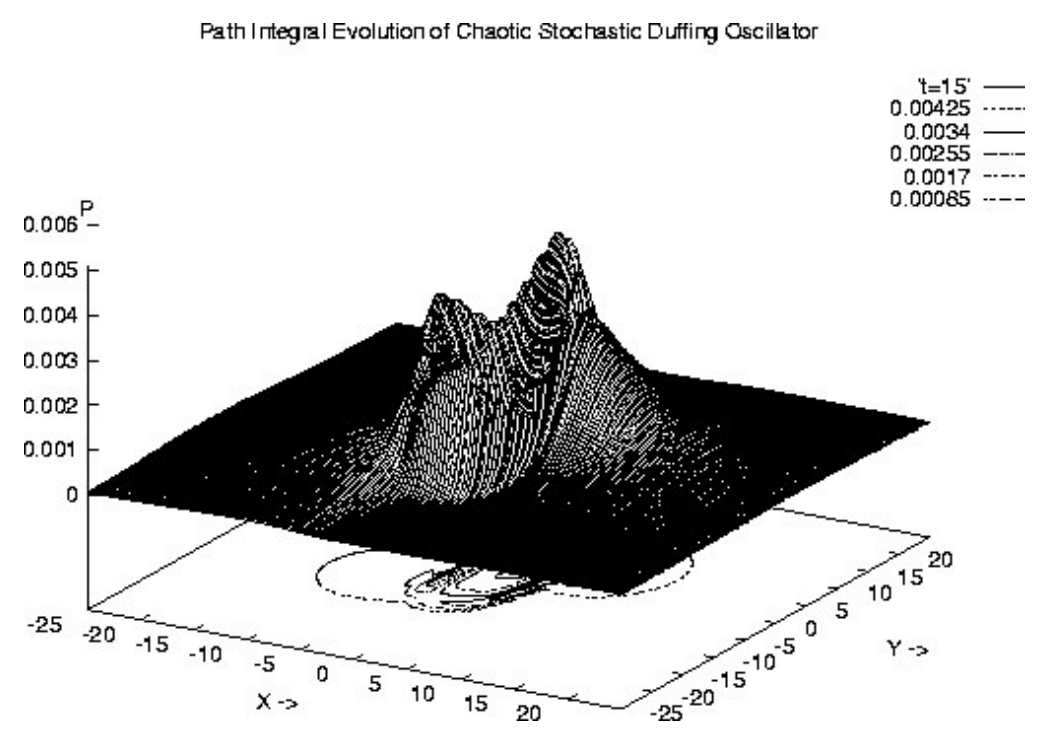

Fig. 2. Illustrated is the evolution of the phase space for a purported model of chaos for the chaotic parameter $\omega_{0}=0.1$ during a transient period between $t=12$ and $t=15.5$, where foldings of the distribution were taken each $d t=0.1$ interval. The non-chaos case with $\omega_{0}=1.0$ is similar in appearance. There is no evidence of chaos (Ingber et al., 1996).

\subsection{Path integral in Stratonovich (midpoint) representation}

The path integral in the Feynman (midpoint) representation enables examination of discretization issues (Langouche et al. 1979,1982 Schulman, 1981). (N.b. $g^{\dagger}$ in DM implies a prepoint evaluation.)

Unless explicitly stated, the Einstein summation convention is used which implies repeated indices signify summation; bars $|\ldots|$ imply no summation.

$$
\begin{gathered}
P\left[M_{t} \mid M_{t_{0}}\right] d M(t)=\int \ldots \int D M \exp \left(-\min \int_{t_{0}}^{t} d t^{\prime} L\right) \delta\left(M\left(t_{0}\right)=M_{0}\right) \delta\left(M(t)=M_{t}\right) \\
D M=\lim _{u \rightarrow \infty} \prod_{\rho=1}^{u+1} g^{\dagger 1 / 2} \prod_{G}(2 \pi \theta)^{-1 / 2} d M_{\rho}^{G} \\
L\left(\dot{M}^{G}, M^{G}, t\right)=\frac{1}{2}\left(\dot{M}^{G}-h^{G}\right) g_{G G^{\prime}}\left(\dot{M}^{G^{\prime}}-h^{G^{\prime}}\right)+\frac{1}{2} h_{; G}^{G}+R / 6-V \\
\dot{M}^{G}(t) \rightarrow M_{\rho+1}^{G}-M_{\rho}^{G}, M^{G}(t) \rightarrow \frac{1}{2}\left(M_{\rho+1}^{G}+M_{\rho}^{G}\right),[\ldots]_{, G}=\frac{\partial[\ldots]}{\partial M^{G}} \\
h^{G}=g^{G}-\frac{1}{2} g^{-1 / 2}\left(g^{1 / 2} g^{G G^{\prime}}\right)_{G^{\prime}}, h_{; G}^{G}=h_{, G}^{G}+\Gamma_{G F}^{F} h^{G}=g^{-1 / 2}\left(g^{1 / 2} h^{G}\right)_{, G} \\
g_{G G^{\prime}}=\left(g^{G G^{\prime}}\right)^{-1}, g=\operatorname{det}\left(g_{G G^{\prime}}\right)
\end{gathered}
$$




$$
\begin{gathered}
\Gamma_{J K}^{F} \equiv g^{L F}[J K, L]=g^{L F}\left(g_{J L, K}+g_{K L, J}-g_{J K, L}\right) \\
R=g^{J L} R_{J L}=g^{J L} g^{J K} R_{F J K L} \\
R_{F J K L}=\frac{1}{2}\left(g_{F K, J L}-g_{J K, F L}-g_{F L, J K}+g_{J L, F K}\right)+g_{M N}\left(\Gamma_{F K}^{M} \Gamma_{J L}^{N}-\Gamma_{F L}^{M} \Gamma_{J K}^{N}\right)
\end{gathered}
$$

Non-constant diffusions add terms to drifts. A Riemannian-curvature potential $R / 6$ is induced for dimension $>1$ in this discretization.

\subsection{Path integral in Ito (prepoint) representation}

In the Ito (prepoint) representation:

$$
\begin{gathered}
P\left[M_{t} \mid M_{t_{0}}\right] d M(t)=\int \ldots \int D M \exp \left(-\min \int_{t_{0}}^{t} d t^{\prime} L\right) \delta\left(M\left(t_{0}\right)=M_{0}\right) \delta\left(M(t)=M_{t}\right) \\
D M=\lim _{u \rightarrow \infty} \prod_{\rho=1}^{u+1} g^{1 / 2} \prod_{G}(2 \pi \Delta t)^{-1 / 2} d M_{\rho}^{G} \\
L\left(\dot{M}^{G}, M^{G}, t\right)=\frac{1}{2}\left(\dot{M}^{G}-g^{G}\right) g_{G G^{\prime}}\left(\dot{M}^{G^{\prime}}-g^{G^{\prime}}\right)+R / 6 \\
\dot{M}^{G}(t) \rightarrow M_{\rho+1}^{G}-M_{\rho}^{G}, M^{G}(t) \rightarrow M_{\rho}^{G} \\
\left(g_{G G^{\prime}}\right)=\left(g^{G G^{\prime}}\right)^{-1}, g=\operatorname{det}\left(g_{G G^{\prime}}\right)
\end{gathered}
$$

The diagonal diffusions are $g^{|G G|}$ and the drifts are $g^{G}$.

\subsection{Path-integral Riemannian geometry}

The midpoint derivation develops a Riemannian geometry with metric defined by the inverse of the covariance matrix

$$
g_{G G^{\prime}}=\left(g^{G G^{\prime}}\right)^{-1}
$$

and where $R$ is the Riemannian curvature

$$
R=g^{J L} R_{J L}=g^{J L} g^{J K} R_{F J K L}
$$

An Ito prepoint discretization for the same probability distribution $P$ gives a simpler algebraic form,

$$
\begin{gathered}
M\left(\bar{t}_{s}\right)=M\left(t_{s}\right) \\
L=\frac{1}{2}\left(d M^{G} / d t-g^{G}\right) g_{G G^{\prime}}\left(d M^{G^{\prime}} / d t-g^{G^{\prime}}\right)-V
\end{gathered}
$$

but the Lagrangian $L$ so specified does not satisfy a variational principle. Its variational principle is only useful in the weak-noise limit. This often means that finer meshes are required. 


\subsection{Three approaches are mathematically equivalent}

Three basic different approaches are mathematically equivalent:

(a) Fokker-Planck/Chapman-Kolmogorov partial-differential equations

(b) Langevin coupled stochastic-differential equations

(c) Lagrangian or Hamiltonian path-integrals

Many researchers are familiar with at least one of these descriptions to complex systems.

The path-integral approach derives intuitive physical variables from the Lagrangian $L$ in terms of underlying variables $M^{G}$ :

$$
\begin{gathered}
\text { Momentum }: \Pi^{G}=\frac{\partial L}{\partial\left(\partial M^{G} / \partial t\right)} \\
\text { Mass : } g_{G G^{\prime}}=\frac{\partial L}{\partial\left(\partial M^{G} / \partial t\right) \partial\left(\partial M^{G^{\prime}} / \partial t\right)} \\
\text { Force }: \frac{\partial L}{\partial M^{G}} \\
F=m a: \delta L=0=\frac{\partial L}{\partial M^{G}}-\frac{\partial}{\partial t} \frac{\partial L}{\partial\left(\partial M^{G} / \partial t\right)}
\end{gathered}
$$

The Momenta $=\Pi^{G}$ are called canonical momenta. They have been used as Canonical Momenta Indicators (CMI) for disciplines described in this paper, and are proposed to be used in the application to COVID-19 forecasting.

Differentiation especially of noisy systems usually introduces more noise. The path-integral often gives superior numerical performance because integration is a smoothing process.

\subsubsection{Stochastic differential equation (SDE)}

The Stratonovich (midpoint discretized) Langevin equations can be analyzed in terms of the Wiener process $d W^{i}$, developed with Gaussian noise $\eta^{i}=d W^{i} / d t$.

$$
\begin{gathered}
d M^{G}=f^{G}(t, M(t)) d t+\hat{g}_{i}^{G}(t, M(t)) d W^{i} \\
\dot{M}^{G}(t)=f^{G}(t, M(t))+\hat{g}_{i}^{G}(t, M(t)) \eta^{i}(t) \\
d W^{i} \rightarrow \eta^{i} d t \\
M=\left\{M^{G} ; G=1, \ldots, \Lambda\right\} \\
\eta=\left\{\eta^{i} ; i=1, \ldots, N\right\} \\
\dot{M}^{G}=d M^{G} / d t \\
<\eta^{j}(t)>_{\eta}=0,<\eta^{j}(t), \eta^{j^{\prime}}\left(t^{\prime}\right)>_{\eta}=\delta^{j j^{\prime}} \delta\left(t-t^{\prime}\right)
\end{gathered}
$$

$\eta^{i}$ represents Gaussian white noise. 


\subsubsection{Partial differential equation (PDE)}

The Fokker-Planck, also called Chapman-Kolmogorov, partial differential equation is:

$$
\begin{gathered}
P_{, t}=\frac{1}{2}\left(g^{G G^{\prime}} P\right)_{, G G^{\prime}}-\left(g^{G} P\right)_{, G}+V P \\
P=<P_{\eta}>_{\eta} \\
g^{G}=f^{G}+\frac{1}{2} \hat{g}_{i}^{G^{\prime}} \hat{g}_{i, G^{\prime}}^{G} \\
g^{G G^{\prime}}=\hat{g}_{i}^{G} \hat{g}_{i}^{G^{\prime}} \\
(\ldots)_{G}=\partial(\ldots) / \partial M^{G}
\end{gathered}
$$

$g^{G}$ replaces $f^{G}$ in the SDE if the Ito (prepoint discretized) calculus is used. If boundary conditions are added as Lagrange multipliers, these may enter as a "potential" $V$ creating a Schrodinger-type equation.

\subsection{PATHINT/qPATHINT code}

qPATHINT is an N-dimensional code that calculates propagation of quantum variables in the presence of shocks. Many real systems propagate with sudden changes of state dependent on time. qPATHINT is based on the classical-physics code, PATHINT, useful in several systems across several disciplines. Applications have been made to Statistical Mechanics of Neocortical Interactions (SMNI) and Statistical Mechanics of Financial Markets (SMFM) (Ingber, 2017a,b c).

To numerically calculate the path integral dependent on serial changes in time, standard Monte Carlo techniques generally are not useful. PATHINT was originally developed for this purpose. The PATHINT C code of about 7500 lines of code using the GCC C-compiler was rewritten to use double complex variables instead of double variables, and further developed for arbitrary $\mathrm{N}$ dimensions, creating qPATHINT. The outline of the code is described for classical or quantum systems, using generic coordinates $q$ (Ingber, 2016a, 2017a,b).

The distribution (probabilities for classical systems, wave-functions for quantum systems) can be numerically approximated by a histogram procedure, developing sums of rectangles of height $P_{i}$ and width $\Delta q^{i}$ at points $q^{i}$.

\subsection{Shocks}

Many systems propagate in the presence of continual "shocks". [q]PATHINT and [q]PATHTREE (a fast binominal code based on PATHINT) have had no problems applying these algorithms.

In SMNI, collisions occur via regenerative $\mathrm{Ca}^{2+}$ waves. Interactions take place between the changing magnetic vector potential $\mathbf{A}$ and highly synchronous neuronal firings that produce this potential.

In SMFM, shocks occur due to future dividends, changes in interest rates, changes in asset distributions, etc. 


\subsection{PATHINT/qPATHINT histograms}

A one-dimensional path-integral in variable $q$ in the prepoint Ito discretization is developed in terms of the kernel/propagator $G$, for each of its intermediate integrals, as

$$
\begin{gathered}
P(q ; t+\Delta t)=\int d q^{\prime}\left[g^{1 / 2}(2 \pi \Delta t)^{-1 / 2} \exp (-L \Delta t)\right] P\left(q^{\prime} ; t\right)=\int d q^{\prime} G\left(q, q^{\prime} ; \Delta t\right) P\left(q^{\prime} ; t\right) \\
P(q ; t)=\sum_{i=1}^{N} \pi\left(q-q^{i}\right) P_{i}(t) \\
\pi\left(q-q^{i}\right)=1,\left(q^{i}-\frac{1}{2} \Delta q^{i-1}\right) \leq q \leq\left(q^{i}+\frac{1}{2} \Delta q^{i}\right) ; 0, \text { otherwise }
\end{gathered}
$$

This yields

$$
\begin{gathered}
P_{i}(t+\Delta t)=T_{i j}(\Delta t) P_{j}(t) \\
T_{i j}(\Delta t)=\frac{2}{\Delta q^{i-1}+\Delta q^{i}} \int_{q^{i}-\Delta q^{i-1 / 2}}^{q^{i}+\Delta q^{i} / 2} d q \int_{q^{j}-\Delta q^{j-1} / 2}^{q^{j}+\Delta q^{j} / 2} d q^{\prime} G\left(q, q^{\prime} ; \Delta t\right)
\end{gathered}
$$

$T_{i j}$ is a banded matrix representing the Gaussian nature of the short-time probability centered about the drift.

Several projects have used this algorithm (Ingber \& Nunez, 1995; Ingber et al., 1996; Ingber \& Wilson, 1999; Wehner \& Wolfer, 1983a,b, 1987). 2-dimensional codes were developed for Statistical Mechanics of Combat (SMC), SMNI and SMFM, e.g., handling boundary conditions (Ingber, 2000; Ingber et al., 1991a; Ingber \& Nunez, 1995).

\subsection{Meshes for [q]PATHINT}

Dependence of $L$ on time $t$ can be included explicitly. The mesh $\Delta q^{i}$ is depends on diagonal elements of the diffusion matrix,

$$
\Delta q^{i} \approx\left(\Delta t g^{|i i|}\right)^{1 / 2}
$$

This constrains dependence of the covariance of each variable to a function of that variable, represented by a rectangular mesh. Since integration is a smoothing process (Ingber, 1990), coarser meshes are used relative to stochastic differential equation(s) (Wehner \& Wolfer, 1983a).

Consideration of contributions to first and second moments develop conditions on the time and variable meshes. $\Delta t$ is measured by the diffusion divided by the square of the drift.

\subsection{Lessons learned from SMFM and SMNI}

SMNI qPATHINT emphasizes the need for broad-banded kernels for oscillatory quantum states.

SMFM PATHTREE, and derived qPATHTREE, are financial options code, offering very fast binary calculations, which can be used for nonlinear time-dependent systems (Ingber et al., 2001 ). In contrast to the PATHINT/qPATHINT code that has been generalized to $\mathrm{N}$ dimensions, currently an SMFM [q]PATHTREE is faster but still only a binary tree with $\mathrm{N}=1$ and cannot be effectively applied to multivariate quantum oscillatory systems (Ingber, 2016a, 2017a,b). 


\section{Statistical Mechanics of Neocortical Interactions (SMNI)}

A paper discusses SMNI in more detail (Ingber, 2018a).

As calculated, quantum $\mathrm{Ca}^{2+}$ interactions contribute to EEG. EEG can arise from largescale neocortical firings during attentional tasks. Very specific calcium ions $\mathrm{Ca}^{2+}$ are considered, only those arising from regenerative calcium waves generated at tripartite neuron-astrocyte-neuron synapses. It is important to note that $\mathrm{Ca}^{2+}$ ions and waves influence many processes in the brain (Ross, 2012), but this study focused on free waves generated at tripartite synapses (Lallouette et al., 2019) because of their calculated direct interactions with large synchronous neuronal firings.

These projects includes quantum influences which most certainly is speculative, but it is testable. It is worth continued examination it until it is theoretically or experimentally proven to be false.

SMNI was developed in 1981, scaling synaptic interactions to neuronal firings, to minicolumnarmacrocolumnar columns of neurons to mesocolumnar dynamics, to columns of neuronal firings, to regional macroscopic sites (Ingber, 1981, 1982, 1983, 1984a, 1985, 1994).

SMNI has calculated agreement/fits with experimental data from properties of short-term memory (STM) (Ingber, 2012b) including capacity (auditory $7 \pm 2$ and visual $4 \pm 2$ ) (Ericsson \& Chase, 1982; Zhang \& Simon, 1985), duration, stability, primacy versus recency rule, Hick's law (Hick, 1952; Ingber, 1999; Jensen, 1987), and interactions within macrocolumns during mental rotation of images (Ingber, 1982, 1983, 1984a, 1985, 1994). SMNI models across neocortical regions fit EEG data (Ingber, 1997a,b, 2012b). 


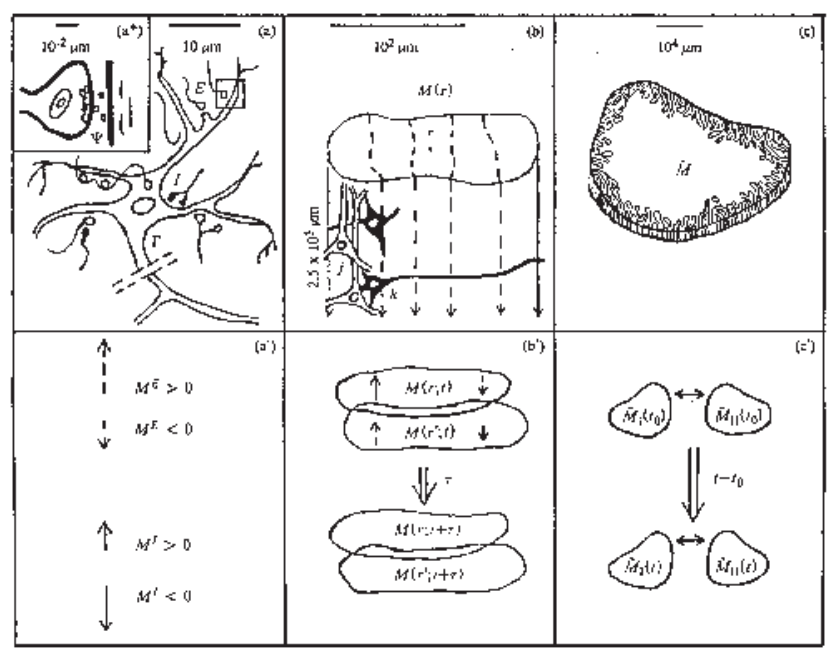

Fig. 3. Illustrated are three SMNI biophysical scales (Ingber, 1982, 1983): (a)-(a*)-(a') microscopic neurons; (b)-(b') mesocolumnar domains; (c)-(c') macroscopic regions. $\left(a^{*}\right)$ : synaptic inter-neuronal interactions at the scale of mesocolumns, described by the mean and variance of a distribution $\Psi$

(a): intraneuronal transmissions described by the mean and variance of $\Gamma$

$\left(a^{\prime}\right)$ : collective mesocolumnar-averaged inhibitory $(I)$ and excitatory $(E)$ neuronal firings $M$

(b): a mesocolumn with its vertical organization of minicolumns with horizontal layers

(b'): overlapping mesocolumns at locations $r$ and $r^{\prime}$ from times $t$ and $t+\tau, \tau$ on the order of $10 \mathrm{msec}$

(c): macroscopic regions of neocortex including many mesocolumnar domains

(c'): regions of neocortex coupled by long-ranged interactions

\subsection{Synaptic interactions}

The short-time conditional probability distribution neuronal firing given just-previous firings of other neurons is derived from chemical and electrical intra-neuronal interactions (Ingber, 1982, 1983). The conditional probability that within $\tau_{j}$ of $5-10 \mathrm{msec}$, a neuron $j$ fires $\left(\sigma_{j}=+1\right)$ or does not fire $\left(\sigma_{j}=-1\right)$ is

$$
\begin{gathered}
p_{\sigma_{j}}=\Gamma \Psi=\frac{\exp \left(-\sigma_{j} F_{j}\right)}{\exp \left(F_{j}\right)+\exp \left(-F_{j}\right)} \\
F_{j}=\frac{V_{j}-\sum_{k} a_{j k}^{*} v_{j k}}{\left(\pi \sum_{k^{\prime}} a_{j k^{\prime}}^{*}\left(v_{j k^{\prime}}^{2}+\phi_{j k^{\prime}}^{2}\right)\right)^{1 / 2}} \\
a_{j k}=\frac{1}{2} A_{|j k|}\left(\sigma_{k}+1\right)+B_{j k}
\end{gathered}
$$

The contribution to polarization achieved at an axon from activity at a synapse is given by $\Gamma$, the "intra-neuronal" probability distribution. $\Psi$ is the "inter-neuronal" probability distribution, of thousands of quanta of neurotransmitters released at one neuron's presynaptic site effecting a (hyper-)polarization at another neuron's postsynaptic site. This holds for $\Gamma$ Poisson, and for $\Psi$ Poisson or Gaussian. 
$V_{j}$ is the depolarization threshold. $v_{j k}$ is the induced synaptic polarization of $E$ or $I$ type at the axon, and $\phi_{j k}$ is its variance. The efficacy $a_{j k}$, related to the impedance across synaptic gaps, is a sum of $A_{j k}$ from the connectivity between neurons, activated if the impinging $k$-neuron fires, and $B_{j k}$ is spontaneous background noise.

\subsection{Neuronal interactions}

Aggregation from microscopic synaptic scales to mesoscopic scales gives the mesoscopic probability $P$

$$
\begin{gathered}
P=\prod_{G} P^{G}\left[M^{G}(r ; t+\tau) \mid M^{\bar{G}}\left(r^{\prime} ; t\right)\right] \\
=\sum_{\sigma_{j}} \delta\left(\sum_{j E} \sigma_{j}-M^{E}(r ; t+\tau)\right) \delta\left(\sum_{j I} \sigma_{j}-M^{I}(r ; t+\tau)\right) \prod_{j}^{N} p_{\sigma_{j}}
\end{gathered}
$$

$M$ represents a mesoscopic scale of columns of $N$ neurons, with subsets $E$ and $I$ represented by $p_{q_{i}}$. The "delta"-functions $\delta$-constraint represents an aggregate of many neurons in a column. $G$ represents excitatory $(E)$ and inhibitory $(I)$ contributions. $\bar{G}$ designates contributions from both $E$ and $I$.

\subsection{Columnar interactions}

In the prepoint (Ito) representation the SMNI Lagrangian $L$ is

$$
\begin{gathered}
L=\sum_{G, G^{\prime}}(2 N)^{-1}\left(\dot{M}^{G}-g^{G}\right) g_{G G^{\prime}}\left(\dot{M}^{G^{\prime}}-g^{G^{\prime}}\right) /(2 N \tau)-V^{\prime} \\
g^{G}=-\tau^{-1}\left(M^{G}+N^{G} \tanh F^{G}\right) \\
g^{G G^{\prime}}=\left(g_{G G^{\prime}}\right)^{-1}=\delta_{G}^{G^{\prime}} \tau^{-1} N^{G} \operatorname{sech}^{2} F^{G} \\
g=\operatorname{det}\left(g_{G G^{\prime}}\right)
\end{gathered}
$$

The threshold factor $F^{G}$ is derived as

$$
\begin{gathered}
F^{G}=\sum_{G^{\prime}} \frac{\nu^{G}+\nu^{\ddagger E^{\prime}}}{\left((\pi / 2)\left[\left(v_{G^{\prime}}^{G}\right)^{2}+\left(\phi_{G^{\prime}}^{G}\right)^{2}\right]\left(\delta^{G}+\delta^{\ddagger E^{\prime}}\right)\right)^{1 / 2}} \\
\nu^{G}=V^{G}-a_{G^{\prime}}^{G} v_{G^{\prime}}^{G} N^{G^{\prime}}-\frac{1}{2} A_{G^{\prime}}^{G} v_{G^{\prime}}^{G} M^{G^{\prime}}, \nu^{\ddagger E^{\prime}}=-a_{E^{\prime}}^{\ddagger E} v_{E^{\prime}}^{E} N^{\ddagger E^{\prime}}-\frac{1}{2} A_{E^{\prime}}^{\ddagger E} v_{E^{\prime}}^{E} M^{\ddagger E^{\prime}} \\
\delta^{G}=a_{G^{\prime}}^{G} N^{G^{\prime}}+\frac{1}{2} A_{G^{\prime}}^{G} M^{G^{\prime}}, \delta^{\ddagger E^{\prime}}=a_{E^{\prime}}^{\ddagger E} N^{\ddagger E^{\prime}}+\frac{1}{2} A_{E^{\prime}}^{\ddagger E} M^{\ddagger E^{\prime}} \\
a_{G^{\prime}}^{G}=\frac{1}{2} A_{G^{\prime}}^{G}+B_{G^{\prime}}^{G}, a_{E^{\prime}}^{\ddagger E}=\frac{1}{2} A_{E^{\prime}}^{\ddagger E}+B_{E^{\prime}}^{\ddagger E}
\end{gathered}
$$

where $A_{G^{\prime}}^{G}$ is the columnar-averaged direct synaptic efficacy, $B_{G^{\prime}}^{G}$ is the columnar-averaged backgroundnoise contribution to synaptic efficacy. The "†" parameters denote regional interactions across many macrocolumns. 


\subsection{SMNI parameters from experiments}

Values of all SMNI parameters are not arbitrarily fit to data, but their bounds are taken from experimental data,

$N^{G}=\left\{N^{E}=160, N^{I}=60\right\}$ are used for visual neocortex, $\left\{N^{E}=80, N^{I}=30\right\}$ are used for other neocortical regions, $M^{G^{\prime}}$ and $N^{G^{\prime}}$ in $F^{G}$ are afferent macrocolumnar firings scaled to efferent minicolumnar firings by $N / N^{*} \approx 10^{-3}$. $N^{*}$ is the number of neurons in a macrocolumn, about $10^{5}$. $V^{\prime}$ includes nearest-neighbor mesocolumnar interactions. $\tau$ considered to be on the order of 5-10 ms.

Other values consistent with experimental data are $V^{G}=10 \mathrm{mV}, v_{G^{\prime}}^{G}=0.1 \mathrm{mV}, \phi_{G^{\prime}}^{G}=$ $0.03^{1 / 2} \mathrm{mV}$.

Nearest-neighbor interactions among columns develop dispersion relations were used to calculate speeds of mental visual rotation (Ingber, 1982, 1983).

The phenomenological wave equation cited by EEG theorists used for fits of SMNI to EEG data (Ingber, 1995) was derived using the variational principle applied to the SMNI Lagrangian (Ingber \& Nunez, 2010).

This permits an audit trail from synaptic parameters to the statistically averaged regional Lagrangian.

\subsection{Verification of basic SMNI hypothesis}

The core SMNI hypothesis developed circa 1980 (Ingber, 1981, 1982, 1983) is that highly synchronous patterns of neuronal firings process high-level information. In 2012 this hypothesis was verified experimentally (Asher, 2012; Salazar et al., 2012).

\subsection{Comparing EEG testing data with training data}

Using EEG data from http://physionet.nlm.nih.gov/pn4/erpbci (Citi et al., 2010; Goldberger et al. 2000), SMNI was fit to P300 synchronous waves during attentional tasks, for each of 12 subjects. It was possible to find 10 Training runs and 10 Testing runs (Ingber, 2016b).

Spline-Laplacian transformations on the EEG potential $\Phi$ were developed from the SMNI $M^{G}$ firing variables at each electrode site. The electric potential $\Phi$ experimentally measured by EEG is not $\mathbf{A}$, but both are derived from the same currents $\mathbf{I}$. A is linearly proportional to $\Phi$ scaled using a parameter fit to data. Additional parameterization of background synaptic parameters $B_{G^{\prime}}^{G}$ and $B_{E^{\prime}}^{\ddagger E}$ modified previous work.

The A model outperformed the no-A model; the no-A model simply used A-non-dependent synaptic parameters. Cost functions with the $|\mathbf{A}|$ model were much worse than either the $\mathbf{A}$ model or the no-A model.

\subsection{PATHINT STM}

A Balanced Centered model (BC) after 500 foldings of $\Delta t=0.01,5$ unit of relaxation time $\tau$, exhibits the existence of ten well developed peaks. These peaks are identified with possible trappings of firing patterns. 


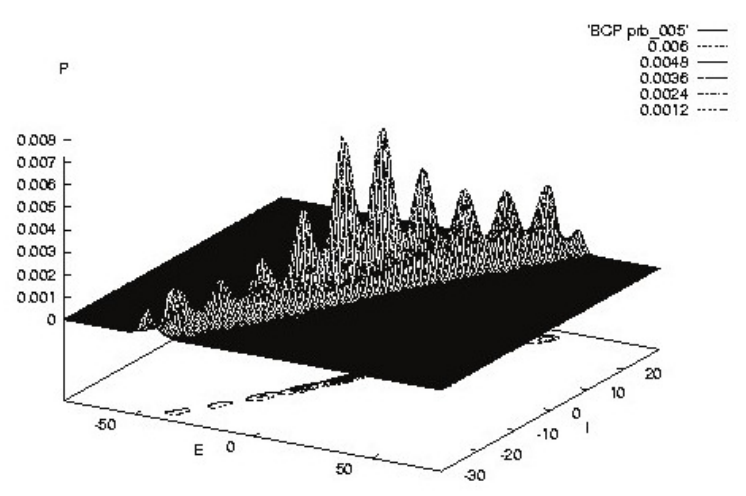

Fig 4. Illustrated is SMNI STM Model BC at the evolution at $5 \tau$ (Ingber \& Nunez, 1995).

This describes the "7 \pm 2 " rule, as calculated by SMNI PATHINT (Ingber \& Nunez, 1995).

\subsection{PATHINT STM visual}

A Balanced Centered Visual model (BCV) with double the number of neurons/minicolumn than other neocortical regions, after 1000 foldings of $\Delta t=0.01,10$ unit of relaxation time $\tau$, exhibits the existence of four well developed peaks. These peaks are identified with possible trappings of firing patterns. Other peaks at lower scales are present, numbering on the same order as in the BC' model, as the strength in the original peaks dissipates throughout firing space, but these smaller peaks are much less probable to be accessed. 


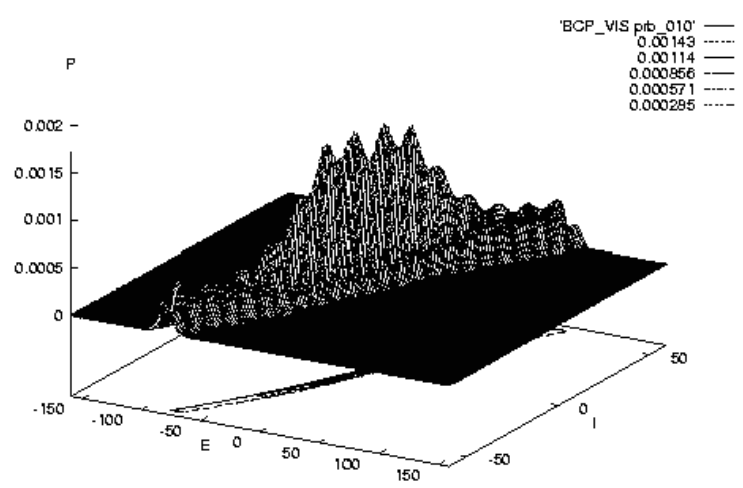

Fig. 5. Illustrated is SMNI STM Model BCV at the evolution at $10 \tau$ (Ingber \& Nunez, 1995).

This describes the " $4 \pm 2$ " rule for visual STM (Ingber \& Nunez, 1995).

\subsection{Tripartite synaptic interactions}

Human brain contains over $10^{11}$ cells, about half of which are neurons; half are glial cells. Astrocytes are a good fraction of glial cells, possibly the majority. Many papers have examines the influence of astrocytes on synaptic processes (Agulhon et al., 2008; Araque \& Navarrete, 2010; Banaclocha et al., 2010; Bellinger, 2005; Innocenti et al., 2000; Pereira \& Furlan, 2009; Reyes \& Parpura, 2009; Scemes \& Giaume, 2006; Volterra et al., 2014).

Glutamate release from astrocytes through a $\mathrm{Ca}^{2+}$-dependent mechanism activates receptors at presynaptic terminals. Intercellular calcium waves (ICWs) travel over hundreds of astrocytes propagating over many neuronal synapses. ICWs contribute to synaptic activity. Glutamate is released in a regenerative manner, with cells involved in a calcium wave releasing additional glutamate (Ross, 2012).

$\left[\mathrm{Ca}^{2+}\right]$ (concentrations of $\mathrm{Ca}^{2+}$ ) affect probabilities at synaptic sites, enhancing release of gliotransmitters.

Free regenerative $\mathrm{Ca}^{2+}$ waves, couple to the magnetic vector potential A produced by highly synchronous collective firings, as measured by EEG.

\subsubsection{Canonical momentum $\Pi=\mathbf{p}+q \mathbf{A}$}

As derived in the Feynman (midpoint) representation of the path integral, canonical momentum $\boldsymbol{\Pi}$ defines the dynamics of a moving particle with momentum $\mathbf{p}$ in an electromagnetic field. In SI units,

$$
\mathbf{\Pi}=\mathbf{p}+q \mathbf{A}
$$

where $q=-2 e$ for $\mathrm{Ca}^{2+}, e$ is the magnitude of the charge of an electron $=1.6 \times 10^{-19} \mathrm{C}$ (Coulomb), and $\mathbf{A}$ is the electromagnetic vector potential. (In Gaussian units $\boldsymbol{\Pi}=\mathbf{p}+q \mathbf{A} / c$, where $c$ is the speed of light.) A represents three components of a 4-vector.

\subsubsection{Vector potential of wire}

A columnar firing state is considered as a wire/neuron with current $\mathbf{I}$ measured in $\mathrm{A}=$ Amperes $=\mathrm{C} / \mathrm{s}$, 


$$
\mathbf{A}(t)=\frac{\mu}{4 \pi} \int \frac{d r}{r} \mathbf{I}
$$

along length $z$ from a perpendicular distance $r$ from a line with thickness $r_{0}$. Neglecting far-field retardation effects yields

$$
\mathbf{A}=\frac{\mu}{4 \pi} \mathbf{I} \log \left(\frac{r}{r_{0}}\right)
$$

where $\mu$ is the magnetic permeability in vacuum $=4 \pi 10^{-7} \mathrm{H} / \mathrm{m}$ (Henry/meter). Note log dependence insensitivity on distance.

A includes contributions from minicolumnar lines of current from hundreds to thousands of macrocolumns, within a region not large enough to include many convolutions, but contributing to large synchronous bursts of EEG.

Electric $\mathbf{E}$ and magnetic $\mathbf{B}$ fields, derivatives of $\mathbf{A}$ with respect to $r$, do not possess logarithmic insensitivity to distance, and therefore do not linearly accumulate strength within and across macrocolumns. Contributions from synchronous firings to P300 measured on the scalp include tens of thousands of macrocolumns spanning 100 to 100's of $\mathrm{cm}^{2}$. Electric fields generated from a minicolumn may fall by half within 5-10 $\mathrm{mm}$, the range of several macrocolumns.

There are other possible sources of magnetic vector potentials not described as wires with currents (Majhi \& Ghosh, 2018). Their net effects are included the vector magnetic potential of net synchronous firings, but not their functional forms.

\subsubsection{Effects of vector potential on momenta}

The momentum $\mathbf{p}$ for a $\mathrm{Ca}^{2+}$ ion with mass $m=6.6 \times 10^{-26} \mathrm{~kg}$, speed on the order of $50 \mu \mathrm{m} / \mathrm{s}$ to $100 \mu \mathrm{m} / \mathrm{s}$, is on the order of $10^{-30} \mathrm{~kg}-\mathrm{m} / \mathrm{s}$. Molar concentrations of $\mathrm{Ca}^{2+}$ waves of tens of thousands of free ions representing about $1 \%$ of a released set, most being buffered, are within a range of about $100 \mu \mathrm{m}$ to about $250 \mu \mathrm{m}$, with a duration of greater than $500 \mathrm{~ms}$, with $\left[\mathrm{Ca}^{2+}\right]$ ranging from $0.1-5 \mu \mathrm{M}\left(\mu \mathrm{M}=10^{-3} \mathrm{~mol} / \mathrm{m}^{3}\right)$.

The magnitude of the current is taken from experimental data on dipole moments $\mathbf{Q}=|\mathbf{I}| \hat{\mathbf{z}}$ where $\hat{\mathbf{z}}$ is the direction of the current $\mathbf{I}$ with the dipole spread over $z$. Estimates of $\mathbf{Q}$ ranges from $1 \mathrm{pA}-\mathrm{m}=10^{-12} \mathrm{~A}-\mathrm{m}$ for a pyramidal neuron (Murakami \& Okada, 2006), to $10^{-9} \mathrm{~A}-\mathrm{m}$ for larger neocortical mass (Nunez \& Srinivasan, 2006). These currents give rise to $q \mathbf{A} \approx 10^{-28} \mathrm{~kg}-\mathrm{m} / \mathrm{s}$. The velocity of a $\mathrm{Ca}^{2+}$ wave can be $\approx 20-50 \mu \mathrm{m} / \mathrm{s}$. In neocortex, a typical $\mathrm{Ca}^{2+}$ wave of 1000 ions, with total mass $m=6.655 \times 10^{-23} \mathrm{~kg}$ times a speed of $\approx 20-50 \mu \mathrm{m} / \mathrm{s}$, gives $\mathbf{p} \approx 10^{-27} \mathrm{~kg}-\mathrm{m} / \mathrm{s}$.

Taking $10^{4}$ synchronous firings in a macrocolumn leads to a dipole moment $|\mathbf{Q}|=10^{-8} \mathrm{~A}-\mathrm{m}$. Taking $z$ to be $10^{2} \mu \mathrm{m}=10^{-4} \mathrm{~m}$, a couple of neocortical layers, giving $|q \mathbf{A}| \approx 2 \times 10^{-19} \times 10^{-7} \times$ $10^{-8} / 10^{-4}=10^{-28} \mathrm{~kg}-\mathrm{m} / \mathrm{s}$,

\subsubsection{Results including quantum scales}

The wave function $\psi_{\mathrm{e}}$ describing the interaction of $\mathbf{A}$ with $\mathbf{p}$ of $\mathrm{Ca}^{2+}$ wave packets was derived analytically (closed form) from the Feynman representation of the path integral using path-integral techniques (Schulten, 1999), modified by the author to include A.

$$
\psi_{\mathrm{e}}(t)=\int d \mathbf{r}_{0} \psi_{0} \psi_{F}=\left[\frac{1-i \hbar t /\left(m \Delta \mathbf{r}^{2}\right)}{1+i \hbar t /\left(m \Delta \mathbf{r}^{2}\right)}\right]^{1 / 4}\left[\pi \Delta \mathbf{r}^{2}\left\{1+\left[\hbar t /\left(m \Delta \mathbf{r}^{2}\right)\right]^{2}\right\}\right]^{-1 / 4}
$$




$$
\begin{gathered}
\times \exp \left[-\frac{\left[\mathbf{r}-\left(\mathbf{p}_{0}+q \mathbf{A}\right) t / m\right]^{2}}{2 \Delta \mathbf{r}^{2}} \frac{1-i \hbar t /\left(m \Delta \mathbf{r}^{2}\right)}{1+\left[\hbar t /\left(m \Delta \mathbf{r}^{2}\right)\right]^{2}}+i \frac{\mathbf{p}_{0} \cdot \mathbf{r}}{\hbar}-i \frac{\left(\mathbf{p}_{0}+q \mathbf{A}\right)^{2} t}{2 \hbar m}\right] \\
\psi_{F}(t)=\int \frac{d \mathbf{p}}{2 \pi \hbar} \exp \left[\frac{i}{\hbar}\left(\mathbf{p}\left(\mathbf{r}-\mathbf{r}_{0}\right)-\frac{\boldsymbol{\Pi}^{2} t}{(2 m)}\right)\right]=\left[\frac{m}{2 \pi i \hbar t}\right]^{1 / 2} \exp \left[\frac{i m\left(\mathbf{r}-\mathbf{r}_{0}-q \mathbf{A} t / m\right)^{2}}{2 \hbar t}-\frac{i(q \mathbf{A})^{2} t}{2 m \hbar}\right] \\
\psi_{0}=\psi\left(\mathbf{r}_{0}, t=0\right)=\left(\frac{1}{\pi \Delta \mathbf{r}^{2}}\right)^{1 / 4} \exp \left(-\frac{\mathbf{r}_{0}^{2}}{2 \Delta \mathbf{r}^{2}}+i \frac{\mathbf{p}_{0} \cdot \mathbf{r}_{0}}{\hbar}\right)
\end{gathered}
$$

$\psi_{0}$ is the initial Gaussian packet, $\psi_{F}$ is the free-wave evolution operator, $\hbar$ is the Planck constant, $q$ is the electronic charge of $\mathrm{Ca}^{2+}$ ions, $m$ is the mass of a wave-packet of $1000 \mathrm{Ca}^{2+}$ ions, $\Delta \mathbf{r}^{2}$ is the spatial variance of the wave-packet, the initial momentum is $\mathbf{p}_{0}$, and the evolving canonical momentum is $\boldsymbol{\Pi}=\mathbf{p}+q \mathbf{A}$. Detailed calculations show that $\mathbf{p}$ of the $\mathrm{Ca}^{2+}$ wave packet and $q \mathbf{A}$ of the EEG field make about equal contributions to $\Pi$ (Ingber, 2015a).

\subsubsection{SMNI $+\mathrm{Ca}^{2+}$ wave-packet}

Tripartite influence on synaptic $B_{G^{\prime}}^{G}$ is measured by the ratio of a wave-packet's $<\mathbf{p}(t)>_{\psi * \psi}$ to $<\mathbf{p}_{0}\left(t_{0}\right)>_{\psi * \psi}$ at the onset of each attentional task. $<>_{\psi * \psi}$ is taken over $\psi_{\mathrm{e}}^{*} \psi_{\mathrm{e}}$.

$$
<\mathbf{p}>_{\psi * \psi}=m \frac{<\mathbf{r}>_{\psi * \psi}}{t-t_{0}}=\frac{q \mathbf{A}+\mathbf{p}_{0}}{m^{1 / 2}|\Delta \mathbf{r}|}\left(\frac{(\hbar t)^{2}+\left(m \Delta \mathbf{r}^{2}\right)^{2}}{\hbar t+m \Delta \mathbf{r}^{2}}\right)^{1 / 2}
$$

A changes slower than $\mathbf{p}$, and so a static approximation of $\mathbf{A}$ to derive $\psi_{\mathrm{e}}$ and $<\mathbf{p}>_{\psi * \psi}$ is reasonable to use within P300 EEG epochs, resetting $t=0$ at the onset of each classical EEG measurement (1.953 ms apart), using current A. This tests of interactions across scales in a classical context. Note the explicit dependence on $\hbar$, permitting testing quantum sensitivity.

\subsubsection{Results using $<\mathbf{p}>_{\psi * \psi}$}

$<\mathbf{p}>_{\psi * \psi}$ was used in classical-physics SMNI fits to EEG data using ASA. Training used ASA with $100 \mathrm{~K}$ generated states over 12 subjects with and without $\mathbf{A}$, followed by 1000 generated states with the simplex local code contained with ASA. Calculations used one additional parameter across all EEG regions to weight the contribution to synaptic background $B_{G^{\prime}}^{G}$. A is taken to be proportional to the currents measured by EEG, i.e., firings $M^{G}$ (Ingber, 1984a).

\subsubsection{Quantum Zeno effects}

The quantum-mechanical wave function of the wave packet "survives" overlaps after multiple collisions, due to regenerative processes during the observed long durations of hundreds of ms. Therefore $\mathrm{Ca}^{2+}$ waves may support a Zeno or "bang-bang" effect which may promote long coherence times (Burgarth et al., 2018; Facchi et al., 2004; Facchi \& Pascazio, 2008; Giacosa \& Pagliara, 2014; Kozlowski et al., 2015; Muller et al., 2016; Patil et al., 2015; Wu et al., 2012).

The Zeno/"bang-bang" effect may exist only in special contexts, since decoherence among particles is known to be very fast, e.g., faster than phase-damping of macroscopic classical particles colliding with quantum particles (Preskill, 2015).

The quantum wave-packet may be perpetuated by collisions of ions as they enter and leave the wave packet due to the regenerative collisions by the Zeno/ "bang-bang" effect. 


\subsubsection{Survival of wave packet}

In momentum space, the wave packet $\phi(\mathbf{p}, t)$ is considered as "kicked" from $\mathbf{p}$ to $\mathbf{p}+\delta \mathbf{p}$. Random repeated kicks of $\delta \mathbf{p}$ result in $\left\langle\delta \mathbf{p}>\approx 0\right.$, and each kick keeps the variance $\Delta(\mathbf{p}+\delta \mathbf{p})^{2} \approx \Delta(\mathbf{p})^{2}$. Then, the overlap integral at the moment $t$ of a kick between the new and old state is

$$
\begin{gathered}
<\phi^{*}(\mathbf{p}+\delta \mathbf{p}, t) \mid \phi(\mathbf{p}, t)>=\exp \left(\frac{i \kappa+\rho}{\sigma}\right) \\
\kappa=8 \delta \mathbf{p} \Delta \mathbf{p}^{2} \hbar m\left(q \mathbf{A}+\mathbf{p}_{0}\right) t-4\left(\delta \mathbf{p} \Delta \mathbf{p}^{2} t\right)^{2} \\
\rho=-(\delta \mathbf{p} \hbar m)^{2} \\
\sigma=8(\Delta \mathbf{p} \hbar m)^{2}
\end{gathered}
$$

where $\phi(\mathbf{p}+\delta \mathbf{p}, t)$ is the normalized wave function in $\mathbf{p}+\delta \mathbf{p}$ momentum space. An estimate of the survival time amplitude $A(t)$ and survival probability $p(t)$ is (Facchi \& Pascazio, 2008),

$$
\begin{gathered}
A(t)=<\phi^{*}(\mathbf{p}+\delta \mathbf{p}, t) \mid \phi(\mathbf{p}, t)> \\
p(t)=|A(t)|^{2}
\end{gathered}
$$

This yields

$$
<\phi^{*}(\mathbf{p}+\delta \mathbf{p}, t) \mid \phi(\mathbf{p}, t)>=\exp \left(i\left(1.67 \times 10^{-1} t-1.15 \times 10^{-2} t^{2}\right)-1.25 \times 10^{-7}\right)
$$

Note that many small repeated kicks do not appreciably affect the real part of $\phi$, and projections do not appreciably destroy the original wave packet, giving a survival probability per kick as $p(t) \approx \exp \left(-2.5 \times 10^{-7}\right) \approx 1-2.5 \times 10^{-7}$.

The time-dependent phase terms are sensitive to times of tenths of a sec. These times are prominent in STM and in synchronous neural firings. Therefore, $\mathbf{A}$ effects on $\mathrm{Ca}^{2+}$ wave functions maximize their influence on STM at frequencies consistent with synchronous EEG during STM.

All calculations support this model, in contrast to other models of quantum brain processes without such calculations (Hagan et al., 2002; Hameroff \& Penrose, 2013; McKemmish et al., 2009).

\subsection{Nano-robotic applications}

Pharmaceutical products may deliver drugs in nanosystems that affect unbuffered $\mathrm{Ca}^{2+}$ waves in neocortex (Ingber, 2015a). A Ca ${ }^{2+}$-wave momentum-sensor would be like a piezoelectric device.

At the onset of a $\mathrm{Ca}^{2+}$ wave (100's of ms), a change of momentum can be about $10^{-30} \mathrm{~kg}-\mathrm{m} / \mathrm{s}$ for a $\mathrm{Ca}^{2+}$ ion. $\mathrm{A} \mathrm{Ca}^{2+}$ wave packet of 1000 ions with onset time of $1 \mathrm{~ms}$, exerts a force on the order of $10^{-24} \mathrm{~N}\left(1 \mathrm{~N} \equiv 1\right.$ Newton $\left.=1 \mathrm{~kg}-\mathrm{m} / \mathrm{s}^{2}\right)$. A nano-robot attracted to this site could deposit chemicals/drugs that interact with the regenerative $\mathrm{Ca}^{2+}$-wave process.

An area of the receptor of the nanosystem of $1 \mathrm{~nm}^{2}$ would require a pressure sensitivity of $10^{-6} \mathrm{~Pa}\left(1 \mathrm{~Pa}=1\right.$ pascal $\left.=1 \mathrm{~N} / \mathrm{m}^{2}\right)$.

The nano-robot could be switched on/off at a regional/columnar level by its sensitivity to local electric/magnetic fields. Highly synchronous firings during STM processes can be affected by these piezoelectric nanosystems via control of $\mathrm{Ca}^{2+}$ waves. In turn, this would affect the influence of of $\mathrm{Ca}^{2+}$ waves via the vector potential $\mathbf{A}$, etc. 


\subsection{Free will}

Possible quantum influences on highly synchronous neuronal firings are relevant to STM to understand connections to consciousness and "Free Will" (FW) (Ingber, 2016a,b).

FW may be established using the Conway-Kochen quantum no-clone "Free Will Theorem" (FWT) (Conway \& Kochen, 2006, 2009).

The essence of FWT is that, since quantum states cannot be cloned, a $\mathrm{Ca}^{2+}$ quantum wavepacket cannot generate a state proven to have previously existed. As explained by the authors (Conway \& Kochen, 2006, 2009), experimenters have choices in selecting measurements, shared by (twinned) particles, including the choice of any random number generator used to aid such choices. Those authors maintain that their proof and description of quantum measurements used is general enough to rule out classical randomness, and that classical determinism cannot be supported by such processes as exist in the quantum world.

\subsection{Affective fits to EEG}

The author also has investigated affective modulation of information processing during attention tasks, using the same ASA and SMNI algorithms that were successful in modeling attention states, generalized to affective states (Ingber, 2020b). While single-electrode results are consistent with other results by other authos, the results are not as good as those using attention data. The data used emotional states measured over durations of several minutes, which do not produce large EEG peaks, e.g., such as those associated with attention states which have fractions of seconds of duration. Thus, the SMNI model had much more difficulty fitting affective data than it had with attention data.

\section{$5 \quad$ Statistical Mechanics of Financial Markets (SMFM)}

\subsection{Trading in risk dimensions (TRD) \& risk-management}

The author's previous work developed two-shell recursive trading systems. An inner-shell of trading indicators is adaptively fit to incoming market data. A parameterized trading-rule outer-shell used the ASA code (Ingber, 1993a) to fit the trading system to historical data. A simple fitting algorithm, usually not requiring ASA, was used for an inner-shell fit.

Trading in Risk Dimensions (TRD) (Ingber, 2005, 2010a), added an additional risk-management middle-shell to create a three-shell algorithm. Portfolio-level distributions of copula-transformed multivariate distributions used ASA to importance-sample weightings of these markets.

TRD processes Training and Testing trading systems on historical data, interacting with RealTime trading platforms at all time resolutions, including high-frequency trading (HFT), minute, daily, and monthly. TRD can be run as an independent executable or called as a function.

TRD flexibly interacts with various trading platforms. The TRD code was used for HFT when the author was a Partner with PION Capital 2011-2013.

\subsubsection{Copula transformation}

Gaussian copulas are developed in TRD (Ingber, 2010a). Other copula distributions are possible, such as Student-t distributions. Alternative distributions can be quite slow because inverse transformations typically are not as quick as for Gaussians. 
Copulas are cited as an important component of risk management (Blanco, 2005), and copulas are regarded as the Basel II standard for credit risk management (Horsewood, 2005). While realtime risk-management for intra-day trading is becoming more popular, most approaches still use simpler VaR measures (Dionne et al., 2006).

The copula approach has been extended to more general distributions (Ibragimon, 2005).

\subsubsection{Transformation to Gaussian marginal distributions}

A Normal Gaussian distribution has the form

$$
p(d y)=\frac{1}{\sqrt{2 \pi}} e^{-\frac{d y^{2}}{2}}
$$

with cumulative distribution

$$
F(d y)=\frac{1}{2}\left[1+\operatorname{erf}\left(\frac{d y}{\sqrt{2}}\right)\right]
$$

where the $\operatorname{erf}()$ function is a tabulated function,

$$
\operatorname{erf}(x)=\frac{2}{\sqrt{\pi}} \int_{0}^{x} d x^{\prime} e^{-x^{\prime 2}}
$$

By setting numerical values of the above two cumulative distributions equal, the $x$ marginal variables are transformed to the $y$ marginal variables.

$$
\begin{gathered}
d y=\sqrt{2} \operatorname{erf}^{-1}(2 F(d x)-1) \\
=\sqrt{2} \operatorname{sgn}(d x-m) \operatorname{erf}^{-1}\left(1-e^{-\frac{|d x-m|}{\chi}}\right)
\end{gathered}
$$

The inverse mapping uses

$$
\begin{gathered}
d y \geq 0 \rightarrow d x-m \geq 0 \\
d y<0 \rightarrow d x-m<0 \\
\operatorname{erf}([.])=-\operatorname{erf}(-[.])
\end{gathered}
$$

yields

$$
d x=m-\operatorname{sgn}(d y) \chi \ln \left[1-\operatorname{erf}\left(\frac{|d y|}{\sqrt{2}}\right)\right]
$$

\subsubsection{Including correlations}

Correlations enter the process defined by the $d y^{i}$ marginal transformed variables as

$$
d y^{i}=\hat{g}^{i} d w_{i}
$$

where $d w_{i}$ is the Wiener Gaussian noise contributing to $d y^{i}$ of market $i$.

A given market's noise $\left(\hat{g}^{i} d w_{i}\right)$ may have contributions from $N$ markets, modeled in terms of $N$ independent Gaussian processes $d z_{k}$, 


$$
\hat{g}^{i} d w_{i}=\sum_{k} \hat{g}_{k}^{i} d z_{k}
$$

The covariance matrix $\left(g^{i j}\right)$ of $y$ variables is

$$
g^{i j}=\sum_{k} \hat{g}_{k}^{i} \hat{g}_{k}^{j}
$$

with inverse matrix, where the "metric," is $\left(g_{i j}\right)$ and determinant of $\left(g^{i j}\right)$ is $g$.

With Gaussian variables the covariance matrix is calculated directly from the transformed data (Malevergne \& Sornette, 2002; Rosenberg \& Schuermann, 2004)

Correlations $\rho^{i j}$ are bilinear combinations of market volatilities

$$
\rho^{i j}=\frac{g^{i j}}{\sqrt{g^{i i} g^{j j}}}
$$

Since the transformation to Gaussian space has defined $g^{i i}=1$, the covariance matrices are identical to the correlation matrices.

The multivariate correlated process $P$ in the $d y$ variables, in terms of Lagrangians $L$ and Actions $A$, is

$$
P(d y) \equiv P\left(d y^{1}, \ldots, d y^{N}\right)=(2 \pi d t)^{-\frac{N}{2}} g^{-\frac{1}{2}} e^{-L d t}
$$

where $d t=1$ above. The Lagrangian $\mathrm{L}$ is given by

$$
L=\frac{1}{2 d t^{2}} \sum_{i j} d y^{i} g_{i j} d y^{j}
$$

The effective action $A_{e f f}$, presenting a "cost function" useful for sampling and optimization, is defined by

$$
\begin{gathered}
P(d y)=e^{-A_{\text {eff }}} \\
A_{\text {eff }}=L d t+\frac{1}{2} \ln g+\frac{N}{2} \ln (2 \pi d t)
\end{gathered}
$$

\subsubsection{Stable covariance matrices}

Covariance matrices can be noisy, so often they must be further filtered. The root cause of this noise is recognized as "volatility of volatility" present in markets, which doubles the number of stochastic variables to consider (Ingber \& Wilson, 1999). In addition ill-conditioned matrices can arise from loss of precision for large variables sets.

A good approach for avoiding ill-conditioning and lack of positive-definite matrices is preaveraging of input data using a window of three epochs (Litterman \& Winkelmann, 1998). Other methods in the literature use subtracting eigenvalues of parameterized random matrices (Laloux et al., 1999). Gaussian transformed data alleviates problems usually encountered with fat-tailed distributions. Selection of reasonable windows, coupled with pre-averaging, seems to robustly avoid ill-conditioning. This was tested in portfolios of 20-100 markets. 


\subsubsection{Copula of multivariate correlated distribution}

The multivariate distribution in $x$-space uses

$$
P(d x)=P(d y)\left|\frac{\partial d y^{i}}{\partial d x^{j}}\right|
$$

where $\left|\frac{\partial d y^{i}}{\partial d x^{j}}\right|$ is the Jacobian matrix of this transformation. This gives

$$
P(d x)=g^{-\frac{1}{2}} e^{-\frac{1}{2} \sum_{i j}\left(d y_{d x}^{i}\right)^{\dagger}\left(g_{i j}-I_{i j}\right)\left(d y_{d x}^{j}\right)} \prod_{i} P_{i}\left(d x^{i}\right)
$$

where $\left(d y_{d x}\right)$ is the column-vector of $\left(d y_{d x}^{1}, \ldots, d y_{d x}^{N}\right)$ expressed back in terms of their respective $\left(d x^{1}, \ldots, d x^{N}\right),\left(d y_{d x}\right)^{\dagger}$ is the transpose row-vector, and $(I)$ is the identity matrix (all ones on the diagonal).

The Gaussian copula $C(d x)$ is defined from Eq. (0),

$$
C(d x)=g^{-\frac{1}{2}} e^{-\frac{1}{2} \sum_{i j}\left(d y_{d x}^{i}\right)^{\dagger}\left(g_{i j}-I_{i j}\right)\left(d y_{d x}^{j}\right)}
$$

\subsection{PATHTREE, PATHINT \& options}

The author has developed a full suite of options codes, which may be integrated with TRD, or used independently.

In the early 1990's the author used PATHINT to evolve multivariate probability distributions in several disciplines. A faster algorithm PATHTREE was created, a binomial tree to evolve such probability distributions. PATHTREE was thoroughly tested and published (Ingber et al., 2001). Both PATHTREE and PATHINT have been applied to options codes, e.g., delivering full sets of Greeks based on such underlying probability distributions. Because of its speed of processing, PATHTREE has been used to fit the shape of distributions to strike data, i.e., a robust bottomup approach to modeling dependence of strikes on volatilities. New codes, qPATHTREE and qPATHINT, have been developed for similar use in quantum complex-variable spaces (Ingber, 2016a, 2017a,b,c, 2018a,b c).

\subsection{Financial options}

The final product, in addition to training use of calculated indicators, is a full set of options Greeks which are necessary indicators for the use of options. For a portfolio $\Pi$ containing an option and its underlying $S$ with volatility $\sigma$, the algebra of "Greeks" is summarized as (Ingber, 2002)

$$
\begin{gathered}
d \Pi=\frac{\partial \Pi}{\partial S} d S+\frac{1}{2} \frac{\partial^{2} \Pi}{\partial d S^{2}} d S^{2}+\frac{\partial \Pi}{\partial \sigma} d \sigma+\frac{\partial \Pi}{\partial t} d t \\
+\frac{\partial^{2} \Pi}{\partial S \partial \sigma} d S d \sigma+\frac{1}{2} \frac{\partial^{2} \Pi}{\partial \sigma^{2}} d \sigma^{2}+\frac{1}{6} \frac{\partial^{3} \Pi}{\partial S^{2} \partial \sigma} d S^{2} d \sigma+\ldots
\end{gathered}
$$

translating to

$$
\begin{gathered}
d \Pi=\Delta d S+\frac{1}{2} \Gamma d S^{2}+K d \sigma+\Theta d t \\
+\Delta^{\prime} d S d \sigma+\frac{1}{2} K^{\prime} d \sigma^{2}+\frac{1}{6} \Gamma^{\prime} d S^{2} d \sigma+\ldots
\end{gathered}
$$




\subsection{Other portfolios}

\subsubsection{Portfolio of physiological indicators (PPI)}

General portfolios of specialized constituents can be addressed, e.g., multiple synchronous imaging data, processed with the TRD copula risk analysis, and using SMNI models (Ingber, 1982, 1983, 2008a). leads to a portfolio of physiological indicators (PPI) to enhance resolution of neocortical processing information (Ingber, 2006a).

\subsubsection{Ideas by statistical mechanics (ISM)}

"Ideas by Statistical Mechanics (ISM)", models evolution and propagation of ideas/patterns throughout populations subjected to endogenous and exogenous interactions (Ingber, 2006b, 2007a, 2008b). This approach offers decision support for projects ranging from diplomatic, information, military, and economic (DIME) factors of propagation/evolution of ideas, to commercial sales, trading indicators across sectors of financial markets, advertising and political campaigns, etc.

\subsubsection{Real options for project schedules (ROPS)}

Similar tools price complex projects as financial options with alternative schedules and strategies. In this project, PATHTREE processes real-world options, including nonlinear distributions and time-dependent starting and stopping of sub-projects, with parameters of shapes of distributions fit using ASA to optimize cost and duration of sub-projects (Ingber, 2007b, 2010b).

\subsection{Developing bid-ask probabilities for high-frequency trading}

This section paraphrases a recent paper (Ingber, 2020a).

High-frequency trading (HFT) is a relatively new development in financial markets, where it has become a primary force in market pricing. A large scientific literature has developed to understand the nature of these forces (Ait-Sahalia \& Saglam, 2017; Avellaneda \& Stoikov, 2008; Baradely et al., 2018; Cartea et al., 2014; Cont, 2011; Cont et al., 2009; Fodra \& Labadie, 2012; Gueant, 2017; Huang et al., 2014).

Since HFT by definition implies fast trading, this has prevented complex sophisticated algorithms from being applied to trading. However, complex algorithms can be developed with parameters optimized by the use of simulated annealing, to produce code that can be used in real time (Ingber, 2020a).

This applies a previously developed statistical mechanics of financial markets (SMFM) (Ingber 1984b, 1990, 1996ab, 2000, 2010a, 2017b; Ingber et al., 2001; Ingber \& Mondescu, 2001, 2003 Ingber et al., 1991b; Ingber \& Wilson, 1999, 2000), to developing bid-ask probabilities to be applied to high-frequency data, using methods of fitting price data or returns data to (a) the distribution and (b) fitting the returns. The returns are also developed into closed-form algebra using the path-integral formalism.

As was true for other disciplines, here too the path-integral methodology is used to develop canonical momenta indicators (CMI) useful to forecast direction and strengths of these variables.

\subsubsection{Forecast code}

Tick data was cleaned on-the-fly in $\mathrm{C}$ code, a practical real-time consideration. The data was volume-weighted, and level-weighted by 0.9 , using 20 levels each of bid and ask; the last level was weighted by $0.9^{20}=0.122$. 
In many HFT markets competitive traders' tactics "game" the book of bids and asks, causing the volume at different levels to be much more volatile than the prices

Both prices $S$ and returns $R$ of bids and asks can be fit. The returns are simply

$$
R(t)=S(t) / S(t-1)
$$

Since the trends quickly swallowed by such markets, and the ASA fits had to work longer for fits with prices than with returns, returns were used for most of the runs.

\subsubsection{Analytic returns}

The path integral formalism possesses an analytic (closed form) calculation of the most probable state (Dekker, 1980),

$$
d M^{G} / d t=g^{G}-g^{1 / 2}\left(g^{-1 / 2} g^{G G^{\prime}}\right)_{, G^{\prime}}
$$

\subsubsection{MAXIMA to $\mathrm{C}$ code}

The 2-factor functional forms, ask as $x^{1}$ and bid as $x^{2}$, are indexed by $j$, without the Einstein convention,

$$
\begin{aligned}
& g^{j}=A_{j}+B_{j} x^{j} \\
& \hat{g}_{j}^{j}=C_{j}+D_{j} x^{j} \\
& \hat{g}_{2}^{1}=E_{1}, \hat{g}_{1}^{2}=E_{2}
\end{aligned}
$$

where parameters $\left\{A_{i}, B_{i}, C_{i}, D_{i}, E_{i}\right\}$ are fit to the data. These simple functional forms are Pade approximates, ratios of polynomials, known to be quite robust functional forms to fit data.

The cost function is the effective Action, including the normalization,

$$
A_{e f f}=L d t+N \log (2 \pi d t) / 2+\log (g) / 2
$$

where this analytic expression for returns can be calculated using the algebraic language Maxima. Expressions can be about 1000 characters (without counting spaces).

It is straightforward to convert Maxima output to Fortran code, but not so easy to covert to C. Previous projects used f2c from netlib.org to compile mixed $\mathrm{C}$ and Fortran code, but here the $\mathrm{C}$ requires powers to use the prefix operator-function pow(), whereas Maxima uses postfix operators. This was circumvented by writing all powers as multiple factors with different names, transforming to Fortran, then renaming these the same.

\subsubsection{Dynamic memory}

Arrays for books of data over a day or comprise 10's of megabytes of memory. Static arrays that size crashes $\mathrm{C}$ code, so this projects used dynamic memory with calloc().

\subsubsection{Sampling code}

It is not necessary to use all tick data to get very good fits. A modification of ASA subroutines randomly samples the data that is volume-weighted and level-weighted. A sample size of $100 \mathrm{~K}$ gave as good results as a sample size of several million for a day of BitMEX data. This random sample was used to ASA-fit cost function calculations that ran over the sample for each generated state. 


\subsubsection{Windows of data}

The distribution at any point depends on at least 2 points of weighted bid-ask data, and accounts for correlations and dynamics in the functional form of the drifts and diffusions.

The best use of this approach uses narrow and wide windows for forecasting (Ingber \& Mondescu, 2001). Although similar to standard practice of using windows of raw data, the result is a different than would be calculated using raw incoming data (also volume-weighted and level-weighted), since this approach includes most probable correlated behavior.

If $A_{\text {eff }}$ is fit, then derived return equations are only accurate to a given $d t$ of about $0.1 \mathrm{sec}$, per the expected mesh used in PATHINT. Instead, returns were directly fit to future return data, typically requiring a few million ASA generated states, by "looking ahead" to the next $d t$ point, PATHINT could be used to fold the short-time distribution for many secs, but this would too slow for HFT.

If returns are fit directly to the data, then any $d t$ ranging from $0.1 \mathrm{sec}$ to $5 \mathrm{sec}$ give the same forecast value for given current data (to 6 significant figures), since a set of fitted drifts and diffusion matrices can have parameters slip from their ideal- $d t$ range to still get good fits at another $d t$. Then the probability calculation at any given point reflects essentially the same distribution.

\subsection{7 dt library}

A $d t$ library is created within a desired range of $d t$ 's that are "reasonably" close to the ideal- $d t$, with multiple ASA fits to return data. This defines a library of probabilities that can be used, yielding a range of choices to be made during real-time HFT.

\subsubsection{Updating parameters}

Fitted parameters are updated overnight with new ASA runs. Alternatively, e.g., if there is a sudden change in context of the market and if sufficient recent data of the new context is available, the fast modified Nelder-Mead simplex code that comes with the ASA code can be used to quickly update the parameters.

\subsubsection{CMI}

The forecast also includes the CMI. However, similar to limitations in fitting $A_{\text {eff }}$, which cannot be fit directly to the returns data for any $d t$, here too the forecast CMI at a different $d t$ than ideal- $d t$ need to have more ASA generated states for additional precision to get stable CMI. The CMI are useful guides on upcoming directions and strengths of bids, ask and midprices.

\subsubsection{Volatility risk}

The risk associated with the probability distributions are calculated from the covariance matrix $g^{G G^{\prime}}$. A quick measure is its determinant $g$.

\subsubsection{Discussion}

A 2-factor probability distribution of bid-ask tick data was developed using Maxima to further develop $\mathrm{C}$ code. Parameters of the 2 -factor drifts and diffusion matrix were fit. Fast calculations of narrow and wide windows of data are processed as required for high-frequency trading. 


\section{Statistical Mechanics of Combat (SMC)}

A paper discusses SMC in more detail (Ingber, 1993b). 


\section{Combat Canonical Momenta}

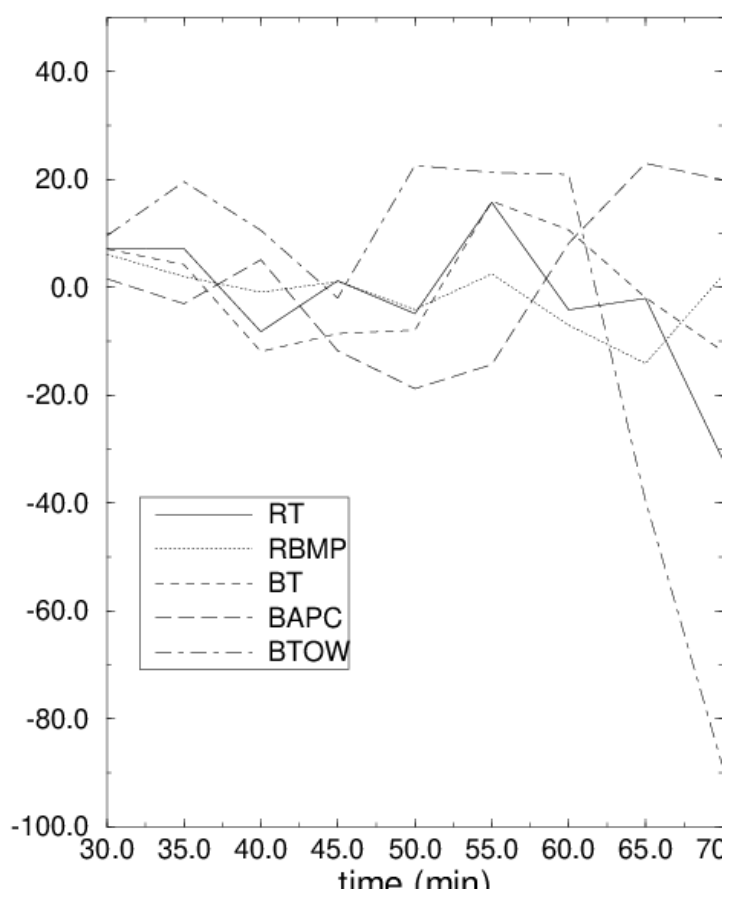

Fig. 6. Upper: The results of Janus(t) attrition of Red and Blue units. Lower: The canonical momenta indicators (CMI) for each system.

\subsection{Necessity of comparing computer models to exercise data}

This project develops a mathematical approach to compare combat exercise data to large-scale computer models wherein men and machines are driven by the natural laws of physics, permitting simulations to be built for training. The focus is to compare the Janus(t) wargame to National Training Center (NTC) data.

"Large scale" here refers to battalion level. (Army systems scale by factors of 3-5, from company to battalion to brigade to division to corps to army.) These battalion level computer models were favorably compared, leading to larger scale corps level, and largest scale theater level computer models. This enhances the value of training on these higher echelon computer models (Bartlett, 1988).

The requirement of depending on combat computer models, including simulations and wargames, has been brought into sharper focus because of: (1) the nonexistence of ample data from previous wars and training operations, (2) the rapidly shortening time scale on which tactical decisions must be made, (3) the rapidly increasing scale at which men and machines are to be deployed, (4) the increasing awareness of new scenarios that are fundamentally different from historical experiences, (5) and the rapidly increasing expense of conducting training exercises. This is as true now in 2020 as it was circa 1990 when this project was developed and employed.

Computer models are used to augment training. Training of these commanders is enhanced if inexpensive pre and post training wargames are provided that statistically replicate training missions. Proper analysis and modeling is required to quantitatively demonstrate that the computer models are good statistical representations of training missions. 


\subsection{Need for data}

High quality simulators are required to augment training in the field, e.g., SIMNET used here, to "validate" the simulator system to some reality.

\subsection{Janus computer simulation of National Training Center \\ 6.4 Description of National Training Center}

The U.S. Army National Training Center (NTC) is located at Fort Irwin, just outside Barstow, California. Circa 1989, there were about $1 / 4$ million soldiers in 80 brigade rotations at NTC, at the level of two battalion task forces (typically about 3500 soldiers and a battalion of 15 attack helicopters), training against two opposing force (OPFOR) battalions resident at NTC. NTC comprised about $2500 \mathrm{~km}^{2}$, and data was collected from battlefield scenarios ranging over about $5 \mathrm{~km}$ linear spread with a maximum lethality range of about $3 \mathrm{~km}$.

Observer-controllers (OC) were present at levels of command down to about platoon level. A rotation can have three force-on-force missions and one live-fire mission. OPFOR platoon and company level personnel are trained US Army soldiers; higher commanders practiced Soviet doctrine and tactics. An OPFOR force typically had carriers (BMPs) and

The primary purpose of data collection during an NTC mission was to develop an after action review (AAR) within a few hours after completion of a mission, giving feedback to commanders. Data from the field, including multiple integrated laser engagement system (MILES) devices, audio communications, OCs, and stationary and mobile video cameras, is sent via relay stations back to a central command center where this was recorded, correlated and abstracted for the AAR. Within a couple of weeks afterwards, a written review was sent to commanders, as part of their NTC take home package.

There were 460 MILES transponders available for tanks for each battle. The B units have transponders, but most did not have transmitters to enable complete pairings of kills targets to be made. Newer MILES devices implemented had transmitters which code their system identification, thereby increasing the number or recordings of pairings. Thus, MILESs without transmitters were not tracked. Man packs with B units enabled most of these men to be tracked, but one man pack could only represent an aggregate of about five people.

B units sent data to A stations (was 48), collected by two C stations atop mountains, and sent through cables to central VAX computers forming a core instrumentation system (CIS). There was a limitation of 400 nodes in computer history for video tracking. About 200 blue and 200 OPFOR units were tracked.

By varying laser intensity and focusing parameters, a maximum laser-beam spread was achieved. A much narrower beam could reach as far as the maximum range. Focusing and attenuation properties of the laser beam made the resolution considerably less than several hundred meters under ideal environmental conditions.

\subsection{Description of Janus(t)}

Janus(t) was an interactive, two-sided, closed, stochastic, ground combat computer simulation. Janus(t) was expanded to include air and naval combat, in several projects with the author's previous thesis students at the Naval Postgraduate School (NPS).

The computer games were interactive. Military analysts made key complex decisions during the simulation, and directly reacted to key actions of the simulated combat forces. Two-sided (Janus was the Greek two-headed god) means that two opposing forces simultaneously were directed by 
two set of players. Closed means that the disposition of the enemy force was not completely known to the friendly forces. Stochastic means that certain events, e.g., the result of a weapon being fired or the impact of an artillery volley, occurred according to laws of chance [random number generators and tables of probabilities of detection (PD), acquisition (PA), hit (PH), kill (PK), etc.]. The principle modeling focus was on those military systems that participate in maneuver and artillery operations. In addition to conventional direct fire and artillery operations, Janus(t) modeled precision guided munitions, minefield employment and breaching, heat stress casualties, suppression, etc.

\subsection{Mathematical formalism}

A typical scenario taken from this NTC study included two red systems, red T-72 tanks $(R T)$ and red armored personnel carriers $(R B M P)$, and three blue systems, blue M1A1 and M60 tanks $(B T)$, blue armored personnel carriers $(B A P C)$, and blue tube-launched optically-tracked wireguided missiles $(B T O W)$, where $R T$ specifies the number of red tanks at a given time $t$, etc. Considered were kills suffered by $B T, \Delta B T$, e.g., within a time epoch $\Delta t \approx 5$ min.

$$
\begin{gathered}
\frac{\Delta B T}{\Delta t} \equiv \dot{B T} \\
=x_{R T}^{B T} R T+y_{R T}^{B T} R T B T+x_{R B M P}^{B T} R B M P+y_{R B M P}^{B T} R B M P B T \\
+z_{B T}^{B T} B T \eta_{B T}^{B T}+z_{R T}^{B T} \eta_{R T}^{B T}+z_{R B M P}^{B T} \eta_{R B M P}^{B T}
\end{gathered}
$$

Here, the $x$ terms represent attrition owing to point fire; the $y$ terms represent attrition owing to area fire. The algebraic forms chosen were consistent with then-current perceptions of aggregated large scale combat. Sources of noise, e.g., arose from PD, PA, PH, PK, etc. Furthermore, such noise had its own functional dependencies, e.g., being proportional to the numbers of units involved in the combat. The $\eta$ represent sources of (white) noise (in the ItThe noise terms were taken to be log normal (multiplicative) noise for the diagonal terms and additive noise for the off-diagonal terms. The diagonal $z$ term $\left(z_{B T}^{B T}\right)$ represents uncertainty associated with the $z$ terms represent uncertainty associated with the $R T$ and $R B M P$. The $x$ and $y$ were constrained such that each term was bounded by the mean of the KVS, averaged over all time and trajectories of similar scenarios; similarly, each $z$ term was constrained to be bounded by the variance of the KVS (Ingber, 1989b). Variables that cannot be so represented, e.g., terrain, C3, weather, etc., were considered as "supervariables" that specified the overall context.

Equations similar to the $\dot{B T}$ equation are also written for $\dot{R T}, R \dot{B M P}, B \dot{A P C}$, and $B T \dot{O} W$. Only $x$ and $y$ that reflect possible nonzero entries in the KVS were used for the fitting procedure. For example, since Janus(t) did not permit direct-fire fratricide, such terms are set to zero. In most NTC scenarios, fratricide typically were a very small effect. Nondiagonal noise terms give rise to correlations in the covariance matrix. Summarizing,

$$
\begin{gathered}
M^{G}=\{R T, R B M P, B T, B A P C, B T O W\} \\
\dot{M}^{G}=g^{G}+\sum_{i} \hat{g}_{i}^{G} \eta^{i} \\
\hat{g}_{i}=z_{i}^{G} M^{G}, i=G \\
\hat{g}_{i}=z_{i}^{G}, i \neq G
\end{gathered}
$$




\subsection{Problems in Lanchester theory}

Many wargames, e.g., joint Theater Level Simulation (JTLS), used such equations as primary algorithms to drive the interactions between opposing forces.

This kind of mathematical modeling was known as Lanchester theory:

$$
\begin{aligned}
& \dot{r}=\mathrm{d} r / \mathrm{d} t=x_{r} b+y_{r} r b \\
& \dot{b}=\mathrm{d} b / \mathrm{d} t=x_{b} r+y_{b} b r
\end{aligned}
$$

where $r$ and $b$ represent red and blue variables, and the $x$ and $y$ are parameters that somehow are fit to actual data.

It is notoriously difficult, if not impossible, to use the simple Lanchester equations to mathematically model any real data with any reasonable degree of precision. These equations were useful to discuss some gross systematics, but it was discouraging to accept that procurement decisions involving billions of dollars of national resources would hinge on mathematical models dependent on Lanchester theory.

Some investigators went further to amass historical data to claim that there was absolutely no foundation for believing that Lanchester theory had anything to do with reality (Dupuy, 1987). This controversy is just one example that supported the necessity of having human intervention in the best of plans, no matter how seemingly sophisticated analysis supports conclusions contrary to human judgment (vanCreveld, 1985).

\subsection{Numerical implementation}

\subsubsection{Fitting parameters}

The five coupled stochastic differential equations, for variables $M^{G}=\{R T, R B M P, B T, B A P C, B T O W\}$, were represented by a short-time conditional probability distribution $P$ in terms of a Lagrangian $L$

$$
P(R \cdot, B \cdot ; t+\Delta t \mid R \cdot, B \cdot ; t)=\frac{1}{(2 \pi \Delta t)^{5 / 2} \sigma^{1 / 2}} \exp (-L \Delta t)
$$

where $\sigma$ is the determinant of the inverse of the covariance matrix, the metric matrix of this space, " $R$." represents $\{R T, R B M P\}$, and " $B$ " represents $\{B T, B A P C, B T O W\}$.

This defined a scalar "dynamic cost function," $C(x, y, z)$,

$$
C(x, y, z)=L \Delta t+\frac{5}{2} \ln (2 \pi \Delta t)+\frac{1}{2} \ln \sigma
$$

which was used with the very fast simulated reannealing (VFSR) algorithm (Ingber, 1989a) to find the (statistically) best fit of $\{x, y, z\}$ to the data.

The form for the Lagrangian $L$ and the determinant of the metric $\sigma$ for the cost function $C$ is

$$
\begin{gathered}
L=\sum_{G} \sum_{G^{\prime}} \frac{\left(\dot{M}^{G}-g^{G}\right)\left(\dot{M}^{G^{\prime}}-g^{G^{\prime}}\right)}{2 g^{G G^{\prime}}} \\
\sigma=\operatorname{det}\left(g_{G G^{\prime}}\right) \\
\left(g_{G G^{\prime}}\right)=\left(g^{G G^{\prime}}\right)^{-1} \\
g^{G G^{\prime}}=\sum_{i} \hat{g}_{i}^{G} \hat{g}_{i}^{G^{\prime}}
\end{gathered}
$$


Generated choices for $\{x, y, z\}$ were constrained by empirical KVSs taken from exercises or from computer simulations of these exercises

$$
\begin{gathered}
g^{G}(t) \leq n^{G}<\Delta M^{G}(t)> \\
\hat{g}_{i}^{G}(t) \leq n_{i}^{G}\left[<\left(\Delta M^{G}(t)\right)^{2}>\right]^{1 / 2}
\end{gathered}
$$

where $n^{G}$ and $n_{i}^{G}$ are the number of terms in $g^{G}$ and $\hat{g}_{i}^{G}$, respectively, and averages $<\cdot>$ are taken over all time epochs and trajectories of similar scenarios.

Since there were competing mathematical forms, it was advantageous to use the path integral to calculate the long-time evolution of $P$ (Ingber, 1989b). Experience demonstrated that, since $P$ is exponentially sensitive to changes in $L$, the long-time correlations derived from theory, measured against the empirical data, was a viable and expedient way of rejecting models not in accord with empirical evidence.

The use of the path integral was a posteriori to the short-time fitting process, and was a subsidiary physical constraint on the mathematical models to judge their internal soundness and suitability for attempts to extrapolate to other scenarios.

\subsubsection{Combat power scores}

After the $\{x, y, z$,$\} were fit to the data and a mathematical model selected, another fit can be$ superimposed to find the effective "combat scores," defined as the relative contribution of each system to the specific class of scenarios. Using a fundamental property of probability distributions, a probability distribution $P_{A}(q)$ of aggregated variables $q_{1}+q_{2}$ was obtained from the probability distribution for $P\left(q_{1}, q_{2}\right)$

$$
P_{A}\left(q=q_{1}+q_{2}\right)=\int \mathrm{d} q_{1} \mathrm{~d} q_{2} P\left(q_{1}, q_{2}\right) \delta\left(q-q_{1}-q_{2}\right)
$$

where $\delta(\cdot)$ is the Dirac delta function.

The aggregated conditional probability was calculated

$$
\begin{gathered}
P_{A}(r, b ; t+\Delta t \mid R \cdot, B \cdot ; t) \\
=\int \mathrm{d} R T \mathrm{~d} R B M P \mathrm{~d} B T \mathrm{~d} B A P C \mathrm{~d} B T O W P(R \cdot, B \cdot ; t+\Delta t \mid R \cdot B \cdot ; t) \\
\times \delta\left(r-w_{R T}^{r} R T-w_{R B M P}^{r} R B M P\right) \\
\times \delta\left(b-w_{R T}^{b} B T-w_{B A P C}^{b} B A P C-w_{B T O W}^{b} B T O W\right)
\end{gathered}
$$

where the $w$ represented the desired combat scores. After the $\{x, y, z\}$ were fitted, the new parameters $\{w\}$ were fit to the data by maximizing the cost function $C^{\prime}(w)$ using VFSR,

$$
C^{\prime}(w)=-\ln P_{A}
$$

It was possible to include human factors in combat power scores, by including human-factors variables in such equations (Ingber \& Sworder, 1991).

\subsubsection{Modeling of National Training Center}

The "kills" attrition data from NTC and the Janus(t)/NTC simulation at once looked strikingly similar during the force-on-force part of the combat. 

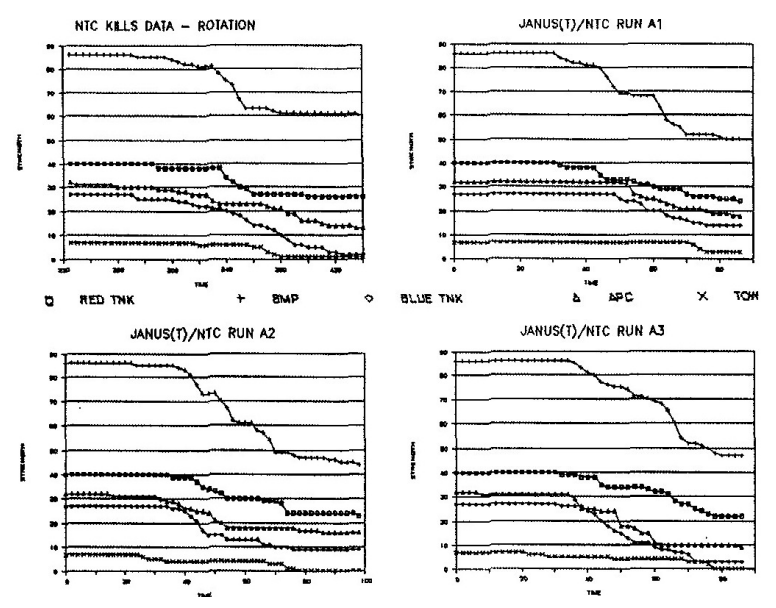

Fig. 7. The upper left box gives NTC vs Janus(t), attrition ("kills") data for an NTC mission. The other 3 boxes give Janus(t) runs using the NTC qualified database.

Note that this fits only half the middle part of the engagement, where the slope of attrition is very steep (and almost linear on the given scale), i.e., the "force on force" part of the engagement. The second half of the data must be predicted by the models.

From the single NTC trajectory qualified, seven 5-min intervals in the middle of the battle were selected. From six Janus(t) runs, similar force-on-force time epochs were identified for a total of 42 data points. In the following fits, $r$ represents red tanks, and $b$ represented blue tanks.

Fitting NTC data to an additive noise model, a cost function of 2.08 gave

$$
\begin{aligned}
& \dot{r}=-2.49 \times 10^{-5} b-4.97 \times 10^{-4} b r+0.320 \eta_{r} \\
& \dot{b}=-2.28 \times 10^{-3} r-3.23 \times 10^{-4} r b+0.303 \eta_{b}
\end{aligned}
$$

Fitting NTC data to a multiplicative noise model, a cost function of 2.16 gave

$$
\begin{aligned}
& \dot{r}=-5.69 \times 10^{-5} b-4.70 \times 10^{-4} b r+1.06 \times 10^{-2}(1+r) \eta_{r} \\
& \dot{b}=-5.70 \times 10^{-4} r-4.17 \times 10^{-4} r b+1.73 \times 10^{-2}(1+b) \eta_{b}
\end{aligned}
$$

Fitting Janus(t) data to an additive noise model, a cost function of 3.53 gave

$$
\begin{aligned}
& \dot{r}=-2.15 \times 10^{-5} b-5.13 \times 10^{-4} b r+0.530 \eta_{r} \\
& \dot{b}=-5.65 \times 10^{-3} r-3.98 \times 10^{-4} r b+0.784 \eta_{b}
\end{aligned}
$$

Fitting Janus(t) data to a multiplicative noise model, a cost function of 3.42 gave

$$
\begin{aligned}
& \dot{r}=-2.81 \times 10^{-4} b-5.04 \times 10^{-4} b r+1.58 \times 10^{-2}(1+r) \eta_{r} \\
& \dot{b}=-3.90 \times 10^{-3} r-5.04 \times 10^{-4} r b+3.58 \times 10^{-2}(1+b) \eta_{b}
\end{aligned}
$$

This comparison illustrates that two different models about equally fit the short-time distribution. The multiplicative noise model showed that approximately a factor of 100 of the noise might be "divided out," or understood in terms of the physical log normal mechanism.

To discern which model best fits the data, the path integral calculation of the long-time distribution was used to see which model best follows the actual data. 


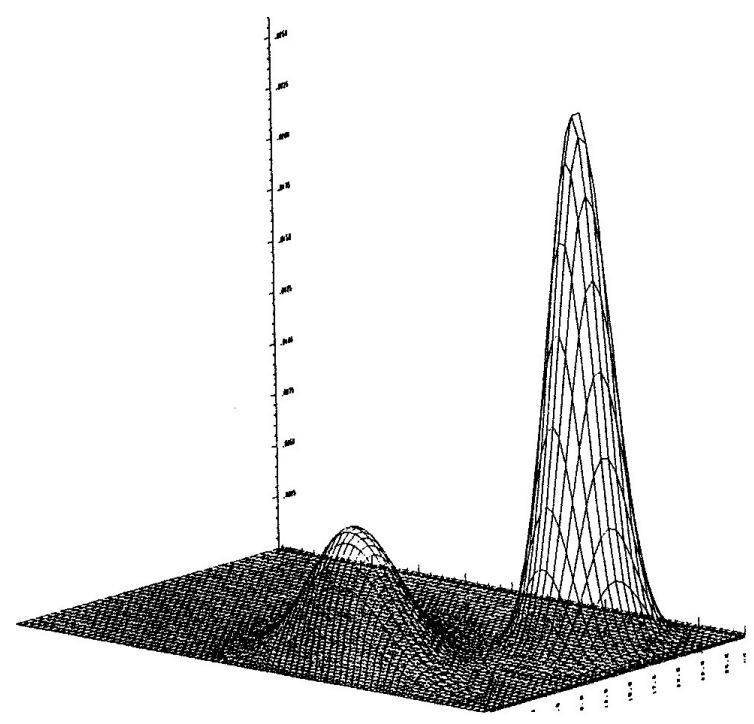

Fig. 8. The path-integral calculation of long-time distribution: Horizontal axes are red (right) and blue (left) forces, for Janus(t) additive noise case, with two superimposed time slices. The initial onset of the engagement was 35 minutes, and peaks represent 50 and 100 minutes.

In general, the probability can be a highly nonlinear algebraic function, and there will be multiple peaks and valleys.

Since only one NTC mission was qualified, the variance of deviation from the mean is not really significant, and given to illustrate that the author's approach could have been applied to more NTC missions as they were qualified and aggregated. Note that only the blue Janus(t) variances of the additive noise model are consistent with the NTC data.

\subsection{Discussion}

Data from 35 to 70 minutes was used for the VFSR-fit. The path integral was used to calculate the long-time evolution of this 5-min distribution from 35 to $70 \mathrm{~min}$. This led to a successful NPS course in simulation for training officer-students.

\section{Applications to COVID-19}

It is generally recognized that the spread of COVID-19 is affected by multiple variables, e.g., potentially including ethnicity, population density, obesity, deprivation, pollution, race, environmental temperature (Anastassopoulou et al., 2020; Bray et al. 2020; Li et al. 2020). Also, the Centre for Evidence-Based Medicine (CEBM) regularly cites papers on the dynamics of COVID-19 at https://www.cebm.net/evidence-synthesis/transmission-dynamics-of-covid-19/ .

This work offers the application of two basic multivariate algorithms to fairly generic issues in forecasting. As such, they may be useful to fit COVID-19 data and to help predict upcoming spread and control of this pandemic.

(a) Adaptive Simulated Annealing (ASA) is an importance-sampling optimization code usually used for nonlinear, nonequilibrium, non-stationary, multivariate systems. 
(b) PATHINT is a numerical path-integral PATHINT code used for propagation of nonlinear probability distributions, including discontinuities.

These codes were developed by the author and applied across multiple disciplines.

There is not "one size fits all" in forecasting different systems. This was demonstrated for three systems (Ingber, 2020c), where the author has addressed multiple projects across multiple disciplines using these tools: 72 papers/reports/lectures in neuroscience, e.g. (Ingber, 2018a, 2020b), 31 papers/reports/lectures in finance, e.g. (Ingber, 2020a; Ingber \& Mondescu, 2003), 24 papers/reports/lectures in combat analyses, e.g. (Ingber, 1993b, 2015b), and 11 papers/reports/lectures in optimization, e.g. (Atiya et al., 2003; Ingber, 2012a), . It is reasonable to expect that this approach can be applied to many other projects.

For example, the path-integral representation of multivariate nonlinear stochastic differential equations permits derivation of canonical momenta indicators (CMI) which are faithful to intuitive concepts like Force, Momenta, Mass, etc (Ingber, 1996a, 2015b; Ingber \& Mondescu, 2001). Correlations among variables are explicitly included in the CMI.

\subsection{Data}

A large and updated database for COVID-19 is maintained by the Centers for Disease Control and Prevention (CDC) at https://www.cdc.gov/library/researchguides/2019novelcoronavirus/researcharticles.html and at https://www.cdc.gov/library/researchguides/2019novelcoronavirus/datastatistics.html .

It is noted that EEG changes are observed by COVID-19 patients (Pasini et al., 2020). EEG may provide a reasonable diagnostic tool.

\subsection{Technical considerations}

If there is not time to process large data sets, then the data can be randomly sampled, e.g., as described in another paper, "Developing bid-ask probabilities for high-frequency trading" (Ingber, 2020a).

If the required forecast is longer than the conditional distribution can sustain, PATHINT/PATHTREE can be used to propagate the distribution.

The dataset should be broken into independent Training and Testing subsets, to test the trained distribution. If this is not possible, e.g., because of data or time limitations, at the least experts can be used to judge if the model is ready for real-time applications, e.g., the Delphi method (Okoli \& Pawlowski, 2004).

If an algorithm like ASA is to be used across a large class of problems, then it must be tunable to different classes. Over the 30+ years of ASA development, the author has worked with many volunteers who have contributed valuable ideas, modifications and corrections to this code. This has resulted in over 150 ASA options that can be used for additional timing additional tuning making it useful across many classes of problems.

The path integral algorithm includes its mathematical equivalents, a large class of stochastic differential equations and a large class of partial differential equations. The advantages of the path integral algorithm are:

(a) intuitive description in terms of classical forces, inertia, momentum, etc., leading to new indicators. 
(b) delivering a cost function derived from a Lagrangian, or its Action (Lagrangian $\mathrm{x}$ dt). Sometimes constraints need to be added as Lagrange multipliers, as was required for normalization requirements in financial risk projects (Ingber, 2010a).

\subsection{Summary}

Two algorithms are suggested for fitting data and forecasting COVID-19, ASA for importancesampling and fitting parameters to models, and PATHINT/PATHTREE. These algorithms have been applied to several disciplines - neuroscience, financial markets, combat analysis. While optimization and path-integral algorithms are now quite well-known (at least to many scientists), these previous applications give strong support to application of these tools to COVID-19 data.

\section{Conclusion}

A basic description of two algorithms is given, ASA for importance-sampling and fitting parameters to models, and PATHINT/PATHTREE (and their quantum analogs qPATHINT/qPATHTREE). Following are three main sections describing forecasting in three quite disparate disciplines - neuroscience, financial markets, combat analysis - using these basic algorithms. While optimization and path-integral algorithms are now quite well-known (at least to many scientists), these applications give strong support to a quite generic application of these tools to stochastic nonlinear systems.

Applications have been described to use these algorithms for forecasting the spread of COVID19, and EEG may provide a reasonable diagnostic tool.

\section{Acknowledgment}

The author thanks the Extreme Science and Engineering Discovery Environment (XSEDE.org), for supercomputer grants since February 2013. The PI has held yearly grants for supercomputer resources from XSEDE, mostly for work on SMNI but for a couple years also to develop qPATHINT and qPATHTREE codes for propagation of quantum wave functions in the presence of random shocks. 


\section{References}

Agulhon, C., Petravicz, J., McMullen, A., Sweger, E., Minton, S., Taves, S., Casper, K., Fiacco, T., McCarthy, K. (2008). What is the role of astrocyte calcium in neurophysiology? Neuron 59, 932-946.

Ait-Sahalia, Y., Saglam, M. (2017). High frequency market making: Optimal quoting. Technical Report SSRN_ID2986523_code758570, Princeton U., Princeton, NJ. http://dx.doi.org/10. 2139/ssrn.2331613.

Anastassopoulou, C., Russo, L., Tsakris, A., Siettos, C. (2020). Data-based analysis, modelling and forecasting of the COVID-19 outbreak. Public Library of Science One 15(3). https://doi. org/10.1371/journal.pone.0230405.

Araque, A., Navarrete, M. (2010). Glial cells in neuronal network function. Philosophical Transactions of The Royal Society B , 2375-2381.

Asher, J. (2012). Brain's code for visual working memory deciphered in monkeys NIH-funded study. Technical Report NIH Press Release, NIH, Bethesda, MD. http://www.nimh.nih.gov/news/ science-news/2012/in-sync-brain-waves-hold-memory-of-objects-just-seen.shtml.

Atiya, A., Parlos, A., Ingber, L. (2003). A reinforcement learning method based on adaptive simulated annealing. In Proceedings International Midwest Symposium on Circuits and Systems (MWCAS), December 2003, IEEE CAS, Cairo, Egypt. 121-124. https://www.ingber.com/ asa03_reinforce.pdf.

Avellaneda, M., Stoikov, S. (2008). High-frequency trading in a limit order book. Quantitative Finance 8(3), 217-224.

Banaclocha, M., Bookkon, I., Banaclocha, H. (2010). Long-term memory in brain magnetite. Medical Hypotheses 74(2), 254-257.

Baradely, N., Bouchardz, B., Evangelista, D., Mounjid, O. (2018). Optimal inventory management and order book modeling. Technical Report arXiv:1802.08135v2 [q-fin.TR], Universite ParisDauphine, Paris.

Bartlett, G. (1988). Battle command training program. Phalanx 21, 18-20.

Bellinger, S. (2005). Modeling calcium wave oscillations in astrocytes. Neurocomputing 65(66), 843-850.

Binder, K., Stauffer, D. (1985). A simple introduction to monte carlo simulations and some specialized topics. In Binder, K. (Ed.), Applications of the Monte Carlo Method in Statistical Physics, Springer-Verlag, Berlin. 1-36.

Blanco, C. (2005). Financial Risk Management: Beyond Normality, Volatility and Correlations. Financial Economics Network, Waltham, MA. http://www.fenews.com/fen46/front-sr/ blanco/blanco.html. 
Bray, I., Gibson, A., White, J. (2020). Coronavirus disease 2019 mortality: a multivariate ecological analysis in relation to ethnicity, population density, obesity, deprivation and pollution. Public Health 185, 261-263. https://doi.org/10.1016/j.puhe.2020.06.056.

Burgarth, D., Facchi, P., Nakazato, H., Pascazio, S., Yuasa, K. (2018). Quantum zeno dynamics from general quantum operations. Technical Report arXiv:1809.09570v1 [quant-ph], Aberystwyth U., Aberystwyth, UK.

Cartea, A., Jaimungal, S., Ricci, J. (2014). Buy low sell high: a high frequency trading perspective. SIAM Journal Financial Mathematics 5(1), 415-444.

Cerny, V. (1982). A thermodynamical approach to the travelling salesman problem: An efficient simulation algorithm. Technical Report Report, Comenius University, Bratislava, Czechoslovakia.

Citi, L., Poli, R., Cinel, C. (2010). Documenting, modelling and exploiting P300 amplitude changes due to variable target delays in Donchin's speller. Journal of Neural Engineering 7(056006), 1-21. https://dx.doi.org/10.1088/1741-2560/7/5/056006.

Cont, R. (2011). Statistical modeling of high-frequency financial data. IEEE Signal Processing $28(5), 16-25$.

Cont, R., Stoikov, S., Talreja, R. (2009). A stochastic model for order book dynamics. Operations Research 58(3), 1-21.

Conway, J., Kochen, S. (2006). The free will theorem. Technical Report arXiv:quant-ph/0604079 [quant-ph], Princeton U, Princeton, NJ.

Conway, J., Kochen, S. (2009). The strong free will theorem. Notices of the American Mathematical Society 56(2), 226-232.

Dekker, H. (1980). On the most probable transition path of a general diffusion process. Physics Letters A 80, 99-101.

Dionne, G., Duchesne, P., Pacurar, M. (2006). Intraday value at risk (IVAR) using tick-by-tick data with application to the toronto stock exchange. Technical Report Report, U de Montreal, Montreal. http://www.fma.org/Stockholm/Papers/dionne_duchesne_pacurar.pdf.

Dupuy, T. (1987). Can we rely on computer combat simulations? Armed Forces Journal August, $58-63$.

Ericsson, K., Chase, W. (1982). Exceptional memory. American Scientist 70, 607-615.

Facchi, P., Lidar, D., Pascazio, S. (2004). Unification of dynamical decoupling and the quantum zeno effect. Physical Review A 69(032314), 1-6.

Facchi, P., Pascazio, S. (2008). Quantum zeno dynamics: mathematical and physical aspects. Journal of Physics A 41(493001), 1-45.

Fodra, P., Labadie, M. (2012). High-frequency market-making with inventory constraints and directional bets. Technical Report arXiv:1206.4810v1 [q-fin.TR], EXQIM, Paris.

Geman, S., Geman, D. (1984). Stochastic relaxation, gibbs distribution and the bayesian restoration in images. IEEE Transactions Pattern Analysis Machine Intelligence 6(6), 721-741. 
Giacosa, G., Pagliara, G. (2014). Quantum zeno effect by general measurements. Physical Review A 052107, 1-5.

Goldberger, A., Amaral, L., Glass, L., Hausdorff, J., Ivanov, P., Mark, R., Mietus, J., Moody, G., Peng, C.K., Stanley, H. (2000). PhysioBank, PhysioToolkit, and PhysioNet: components of a new research resource for complex physiologic signals. Circulation 101(23), e215-e220. http://circ.ahajournals.org/cgi/content/full/101/23/e215.

Gueant, O. (2017). Optimal market making. Technical Report arXiv:1605.01862v5 [q-fin.TR], Universite Paris, Paris.

Hagan, S., Hameroff, S., Tuszynski, J. (2002). Quantum computation in brain microtubules: Decoherence and biological feasibility. Physical Review E 65(061901), 1-11. http://link.aps.org/ doi/10.1103/PhysRevE.65.061901.

Hameroff, S., Penrose, R. (2013). Consciousness in the universe: A review of the 'Orch OR' theory. Physics of Life Reviews 403, 1-40. https://dx.doi.org/10.1016/j.plrev.2013.08.002.

Hick, W. (1952). On the rate of gains of information. Quarterly Journal Experimental Psychology $34(4), 1-33$.

Horsewood, R. (2005). Credit Correlation: Maths of All Angles. Financial Economics Network, Waltham, MA. http://www.fenews.com/fen39/euro_angles/euro_angles.html.

Huang, W., Lehalle, C.A., Rosenbaum, M. (2014). Simulating and analyzing order book data: The queue-reactive model. Technical Report arXiv:1312.0563v2 [q-fin.TR], University Pierre et Marie Curie, Paris.

Ibragimon, R. (2005). Copula-based dependence characterizations and modeling for time series. Technical Report Report No. 2094, Harvard Institute Economic Research, Cambridge, MA. http: //post.economics.harvard.edu/hier/2005papers/HIER2094.pdf.

Ingber, L. (1981). Towards a unified brain theory. Journal Social Biological Structures 4, 211-224. https://www.ingber.com/smni81_unified.pdf.

Ingber, L. (1982). Statistical mechanics of neocortical interactions. i. basic formulation. Physica $D$ 5, 83-107. https://www.ingber.com/smni82_basic.pdf.

Ingber, L. (1983). Statistical mechanics of neocortical interactions. dynamics of synaptic modification. Physical Review A 28, 395-416. https://www.ingber.com/smni83_dynamics.pdf.

Ingber, L. (1984a). Statistical mechanics of neocortical interactions. derivation of short-termmemory capacity. Physical Review A 29, 3346-3358. https://www.ingber.com/smni84_stm. pdf.

Ingber, L. (1984b). Statistical mechanics of nonlinear nonequilibrium financial markets. Mathematical Modelling 5(6), 343-361. https://www.ingber.com/markets84_statmech.pdf.

Ingber, L. (1985). Statistical mechanics of neocortical interactions: Stability and duration of the 7+-2 rule of short-term-memory capacity. Physical Review A 31, 1183-1186. https://www. ingber.com/smni85_stm.pdf.

Ingber, L. (1989a). Very fast simulated re-annealing. Mathematical Computer Modelling 12(8), 967-973. https://www.ingber.com/asa89_vfsr.pdf. 
Ingber, L. (1989b). Mathematical comparison of JANUS(T) simulation to national training center. In Johnson, S., Levis, A. (Eds.), The Science of Command and Control: Part II, Coping With Complexity, AFCEA International, Washington, DC. 165-176.

Ingber, L. (1990). Statistical mechanical aids to calculating term structure models. Physical Review A 42(12), 7057-7064. https://www.ingber.com/markets90_interest.pdf.

Ingber, L. (1993a). Adaptive simulated annealing (ASA). Technical Report Global optimization C-code, Caltech Alumni Association, Pasadena, CA. https://www.ingber.com/\#ASA-CODE.

Ingber, L. (1993b). Statistical mechanics of combat and extensions. In Jones, C. (Ed.), Toward a Science of Command, Control, and Communications, American Institute of Aeronautics and Astronautics, Washington, D.C. 117-149. ISBN 1-56347-068-3. https://www.ingber.com/ combat93_c3sci.pdf

Ingber, L. (1994). Statistical mechanics of neocortical interactions: Path-integral evolution of shortterm memory. Physical Review E 49(5B), 4652-4664. https://www.ingber.com/smni94_stm. pdf.

Ingber, L. (1995). Statistical mechanics of multiple scales of neocortical interactions. In Nunez, P. (Ed.), Neocortical Dynamics and Human EEG Rhythms, Oxford University Press, New York, NY. 628-681. ISBN 0-19-505728-7. https://www.ingber.com/smni95_scales.pdf.

Ingber, L. (1996a). Canonical momenta indicators of financial markets and neocortical EEG. In Amari, S.I., Xu, L., King, I., Leung, K.S. (Eds.), Progress in Neural Information Processing, Springer, New York. 777-784. Invited paper to the 1996 International Conference on Neural Information Processing (ICONIP'96), Hong Kong, 24-27 September 1996. ISBN 981-3083-05-0. https://www.ingber.com/markets96_momenta.pdf.

Ingber, L. (1996b). Statistical mechanics of nonlinear nonequilibrium financial markets: Applications to optimized trading. Mathematical Computer Modelling 23(7), 101-121. https: //www. ingber.com/markets96_trading.pdf.

Ingber, L. (1997a). Statistical mechanics of neocortical interactions: Applications of canonical momenta indicators to electroencephalography. Physical Review E 55(4), 4578-4593. https: //www.ingber.com/smni97_cmi.pdf.

Ingber, L. (1997b). EEG Database. UCI Machine Learning Repository, Irvine, CA. http:// archive.ics.uci.edu/ml/datasets/EEG+Database.

Ingber, L. (1999). Statistical mechanics of neocortical interactions: Reaction time correlates of the g factor. Psycholoquy 10(068). Invited commentary on The g Factor: The Science of Mental Ability by Arthur Jensen. http://www.cogsci.ecs.soton.ac.uk/cgi/psyc/newpsy?10.068.

Ingber, L. (2000). High-resolution path-integral development of financial options. Physica A 283(34), 529-558. https://www.ingber.com/markets00_highres.pdf.

Ingber, L. (2002). Statistical mechanics of portfolios of options. Technical Report Report 2002:SMPO, Physical Studies Institute, Chicago, IL. https://www.ingber.com/markets02_ portfolio.pdf.

Ingber, L. (2005). Trading in risk dimensions (TRD). Technical Report Report 2005:TRD, Physical Studies Institute, Ashland, OR. https://www.ingber.com/markets05_trd.pdf. 
Ingber, L. (2006a). Statistical mechanics of neocortical interactions: Portfolio of physiological indicators. Technical Report Report 2006:PPI, Physical Studies Institute, Ashland, OR. https: //www.ingber.com/smni06_ppi.pdf.

Ingber, L. (2006b). Ideas by statistical mechanics (ISM). Technical Report Report 2006:ISM, Physical Studies Institute, Ashland, OR. https://www.ingber.com/smni06_ism.pdf.

Ingber, L. (2007a). Ideas by statistical mechanics (ISM). Journal Integrated Systems Design and Process Science 11(3), 31-54. https://dl.acm.org/doi/abs/10.5555/1517398.1517402.

Ingber, L. (2007b). Real options for project schedules (ROPS). Technical Report Report 2007:ROPS, Physical Studies Institute, Ashland, OR. https://www.ingber.com/markets07_ rops.pdf.

Ingber, L. (2008a). Statistical mechanics of neocortical interactions (SMNI): testing theories with multiple imaging data. NeuroQuantology Journal 6(2), 97-104. Invitedpaper.https://www. ingber.com/smni08_tt.pdf.

Ingber, L. (2008b). AI and ideas by statistical mechanics (ISM). In Rabunal, J., Dorado, J., Pazos, A. (Eds.), Encyclopedia of Artificial Intelligence, Information Science Reference, New York. 58-64. ISBN 978-1-59904-849-9. https://www.igi-global.com/chapter/ ideas-statistical-mechanics/10226.

Ingber, L. (2010a). Trading in risk dimensions. In Gregoriou, G. (Ed.), The Handbook of Trading: Strategies for Navigating and Profiting from Currency, Bond, and Stock Markets, McGraw-Hill, New York. 287-300.

Ingber, L. (2010b). Real options for project schedules (ROPS). SGI Reflections International Journal of Science, Technology $\&$ Management 2(2), 15-20. Invited paper. http://sgi.ac.in/ colleges/newsletters/1146080820111637301.pdf.

Ingber, L. (2012a). Adaptive simulated annealing. In Oliveira, Jr., H., Petraglia, A., Ingber, L., Machado, M., Petraglia, M. (Eds.), Stochastic global optimization and its applications with fuzzy adaptive simulated annealing, Springer, New York. 33-61. Invited Paper. https://www.ingber. com/asa11_options.pdf.

Ingber, L. (2012b). Columnar EEG magnetic influences on molecular development of short-term memory. In Kalivas, G., Petralia, S. (Eds.), Short-Term Memory: New Research, Nova, Hauppauge, NY. 37-72. Invited Paper. https://www.ingber.com/smni11_stm_scales.pdf.

Ingber, L. (2015a). Calculating consciousness correlates at multiple scales of neocortical interactions. In Costa, A., Villalba, E. (Eds.), Horizons in Neuroscience Research, Nova, Hauppauge, NY. 153-186. ISBN 978-1-63482-632-7. Invited paper. https://www . ingber.com/smni15_calc_ conscious.pdf.

Ingber, L. (2015b). Biological impact on military intelligence: Application or metaphor? International Journal of Intelligent Defence Support Systems 5(3), 173-185. https://www.ingber. com/combat15_milint.pdf.

Ingber, L. (2016a). Path-integral quantum PATHTREE and PATHINT algorithms. International Journal of Innovative Research in Information Security 3(5), 1-15. https://www.ingber.com/ path16_quantum_path.pdf. 
Ingber, L. (2016b). Statistical mechanics of neocortical interactions: Large-scale EEG influences on molecular processes. Journal of Theoretical Biology 395, 144-152. https://doi.org/10.1016/ j.jtbi.2016.02.003.

Ingber, L. (2017a). Evolution of regenerative ca-ion wave-packet in neuronal-firing fields: Quantum path-integral with serial shocks. International Journal of Innovative Research in Information Security 4(2), 14-22. https://www.ingber.com/path17_quantum_pathint_shocks.pdf.

Ingber, L. (2017b). Options on quantum money: Quantum path-integral with serial shocks. International Journal of Innovative Research in Information Security 4(2), 7-13. https: //www.ingber.com/path17_quantum_options_shocks.pdf.

Ingber, L. (2017c). Quantum path-integral qPATHINT algorithm. The Open Cybernetics Systemics Journal 11, 119-133. https://doi.org/10.2174/1874110X01711010119.

Ingber, L. (2018a). Quantum calcium-ion interactions with EEG. Sci 1(7), 1-21. https://doi. org/10.3390/sci1010020

Ingber, L. (2018b). Quantum variables in finance and neuroscience. Technical Report Lecture Plates 2018:QVFN, Physical Studies Institute, Ashland, OR. https://www.ingber.com/ path18_qpathint_lecture.pdf.

Ingber, L. (2018c). Quantum variables in finance and neuroscience II. Technical Report Report 2018:FNQV, Physical Studies Institute, Ashland, OR. https://www.ingber.com/path18_ qpathint.pdf.

Ingber, L. (2019). Editorial: Model of models (MOM). Acta Scientific Computer Sciences 2(1), 21-22. https://actascientific.com/ASCS/pdf/model-of-models-mom.pdf.

Ingber, L. (2020a). Developing bid-ask probabilities for high-frequency trading. Virtual Economics 3(2), 7-24. https://doi.org/10.34021/ve.2020.03.02(1).

Ingber, L. (2020b). Quantum calcium-ion affective influences measured by EEG. Technical Report Report 2020:QCIA, Physical Studies Institute, Ashland, OR. https://doi.org/10.20944/ preprints202009.0591.v1.

Ingber, L. (2020c). Forecasting with importance-sampling and path-integrals: Applications to COVID-19. Technical Report Report 2020:FISPI, Physical Studies Institute, Ashland, OR. https://doi.org/10.20944/preprints202009.0385.v2.

Ingber, L., Chen, C., Mondescu, R., Muzzall, D., Renedo, M. (2001). Probability tree algorithm for general diffusion processes. Physical Review E 64(5), 056702-056707. https://www. ingber. com/path01_pathtree.pdf.

Ingber, L., Fujio, H., Wehner, M. (1991a). Mathematical comparison of combat computer models to exercise data. Mathematical Computer Modelling 15(1), 65-90. https://www.ingber.com/ combat91_data.pdf.

Ingber, L., Mondescu, R. (2001). Optimization of trading physics models of markets. IEEE Transactions Neural Networks 12(4), 776-790. Invited paper for special issue on Neural Networks in Financial Engineering. https://www.ingber.com/markets01_optim_trading.pdf. 
Ingber, L., Mondescu, R. (2003). Automated internet trading based on optimized physics models of markets. In Howlett, R., Ichalkaranje, N., Jain, L., Tonfoni, G. (Eds.), Intelligent InternetBased Information Processing Systems, World Scientific, Singapore. 305-356. Invited paper. https://www.ingber.com/markets03_automated.pdf.

Ingber, L., Nunez, P. (1995). Statistical mechanics of neocortical interactions: High resolution path-integral calculation of short-term memory. Physical Review E 51(5), 5074-5083. https: //www.ingber.com/smni95_stm.pdf.

Ingber, L., Nunez, P. (2010). Neocortical dynamics at multiple scales: EEG standing waves, statistical mechanics, and physical analogs. Mathematical Biosciences 229, 160-173. https: //www.ingber.com/smni10_multiple_scales.pdf.

Ingber, L., Srinivasan, R., Nunez, P. (1996). Path-integral evolution of chaos embedded in noise: Duffing neocortical analog. Mathematical Computer Modelling 23(3), 43-53. https://www. ingber.com/path96_duffing.pdf.

Ingber, L., Sworder, D. (1991). Statistical mechanics of combat with human factors. Mathematical Computer Modelling 15(11), 99-127. https://www.ingber.com/combat91_human.pdf.

Ingber, L., Wehner, M., Jabbour, G., Barnhill, T. (1991b). Application of statistical mechanics methodology to term-structure bond-pricing models. Mathematical Computer Modelling 15(11), 77-98. https://www.ingber.com/markets91_interest.pdf.

Ingber, L., Wilson, J. (1999). Volatility of volatility of financial markets. Mathematical Computer Modelling 29(5), 39-57. https://www.ingber.com/markets99_vol.pdf.

Ingber, L., Wilson, J. (2000). Statistical mechanics of financial markets: Exponential modifications to black-scholes. Mathematical Computer Modelling 31(8/9), 167-192. https://www.ingber. com/markets00_exp.pdf.

Innocenti, B., Parpura, V., Haydon, P. (2000). Imaging extracellular waves of glutamate during calcium signaling in cultured astrocytes. Journal of Neuroscience 20(5), 1800-1808.

Jensen, A. (1987). Individual differences in the hick paradigm. In Vernon, P. (Ed.), Speed of Information-Processing and Intelligence, Ablex, Norwood, NJ. 101-175.

Kirkpatrick, S., Gelatt, C., Vecchi, M. (1983). Optimization by simulated annealing. Science 220(4598), 671-680.

Kozlowski, W., Caballero-Benitez, S., Mekhov, I. (2015). Non-hermitian dynamics in the quantum zeno limit. Technical Report arXiv:1510.04857 [quant-ph], U Oxford, Oxford, UK.

Lallouette, J., Pitta, M.D., Berry, H. (2019). Astrocyte networks and intercellular calcium propagation. In Computational Glioscience, Springer, Cham, Switzerland. 177-210. https: //dx.doi.org/10.1007/978-3-030-00817-8_7.

Laloux, L., Cizeau, P., Bouchaud, J., Potters, M. (1999). Noise dressing of financial correlation matrices. Physical Review Letters 83, 1467-1470.

Langouche, F., Roekaerts, D., Tirapegui, E. (1979). Discretization problems of functional integrals in phase space. Physical Review D 20, 419-432. 
Langouche, F., Roekaerts, D., Tirapegui, E. (1982). Functional Integration and Semiclassical Expansions. Reidel, Dordrecht, The Netherlands.

Li, A., Hannah, T., Durbin, J., Dreher, N., McAuley, F., Marayati, N.F., Spiera, Z., Ali, M., Gometz, A., Kostman, J., Choudhri, T. (2020). Multivariate analysis of black race and environmental temperature on COVID-19 in the US. The American Journal of the Medical Sciences 360(4), 348-356. https://doi.org/10.1016/j.amjms.2020.06.015.

Litterman, R., Winkelmann, K. (1998). Estimating covariance matrices. Technical Report Report, Goldman Sachs, New York.

Ma, S. (1985). Statistical Mechanics. World Scientific, Philadelphia.

Majhi, S., Ghosh, D. (2018). Alternating chimeras in networks of ephaptically coupled bursting neurons. Chaos 28(083113). https://doi.org/10.1063/1.5022612.

Malevergne, Y., Sornette, D. (2002). General framework for a portfolio theory with non-gaussian risks and non-linear correlations. Technical Report Report, UCLA, Los Angeles, CA.

Mathews, J., Walker, R. (1970). Mathematical Methods of Physics, 2nd ed. Benjamin, New York, NY.

McKemmish, L., Reimers, J., McKenzie, R., Mark, A., Hush, N. (2009). Penrose-hameroff orchestrated objective-reduction proposal for human consciousness is not biologically feasible. Physical Review E 80(021912), 1-6. http://link.aps.org/doi/10.1103/PhysRevE.80.021912.

Metropolis, N., Rosenbluth, A., Rosenbluth, M., Teller, A., Teller, E. (1953). Equation of state calculations by fast computing machines. Journal of Chemical Physics 21(6), 1087-1092.

Muller, M., Gherardini, S., Caruso, F. (2016). Quantum zeno dynamics through stochastic protocols. Technical Report arXiv:1607.08871v1 [quant-ph], U Florence, Florence, Italy.

Murakami, S., Okada, Y. (2006). Contributions of principal neocortical neurons to magnetoencephalography and electroencephalography signals. Journal of Physiology 575(3), 925-936.

Nunez, P., Srinivasan, R. (2006). Electric Fields of the Brain: The Neurophysics of EEG, 2nd Ed. Oxford University Press, London.

Okoli, C., Pawlowski, S. (2004). The delphi method as a research tool: an example, design considerations and applications. Information and Management 42(1), 15-29. https: //doi.org/10.1016/j.im.2003.11.002.

Oliveira, Jr., H., Petraglia, A., Ingber, L., Machado, M., Petraglia, M. (2012). Stochastic global optimization and its applications with fuzzy adaptive simulated annealing. Springer, New York. https://doi.org/10.1007/978-3-642-27479-4.

Pasini, E., Bisulli, F., Volpi, L., Minardi, I., Tappata, M., Muccioli, L., Pensato, U., Riguzzi, P., Tinuper, P., Michelucci, R. (2020). EEG findings in COVID-19 related encephalopathy. Clinical Neurophysiology 131(9), 2265-2267. https://doi.org/10.1016/j.clinph.2020.07.003.

Patil, Y., Chakram, S., Vengalattore, M. (2015). Measurement-induced localization of an ultracold lattice gas. Physical Review Letters 115(140402), 1-5. http://link.aps.org/doi/10.1103/ PhysRevLett.115.140402. 
Pereira, Jr., A., Furlan, F. (2009). On the role of synchrony for neuron-astrocyte interactions and perceptual conscious processing. Journal of Biological Physics 35(4), 465-480.

Pincus, M. (1970). A monte carlo method for the approximate solution of certain types of constrained optimization problems. Operations Research 18, 1225-1228.

Preskill, J. (2015). Quantum mechanics. Technical Report Lecture Notes, Caltech, Pasadena, CA. http://www.theory.caltech.edu/people/preskill/ph219/.

Reyes, R., Parpura, V. (2009). The trinity of Ca2+ sources for the exocytotic glutamate release from astrocytes. Neurochemistry International 55(3), 1-14.

Rosenberg, J., Schuermann, T. (2004). A general approach to integrated risk management with skewed, fat-tailed risks. Technical Report Staff Report No. 185, Fed. Res. Bank NY, New York, NY.

Ross, W. (2012). Understanding calcium waves and sparks in central neurons. Nature Reviews Neuroscience 13, 157-168.

Salazar, R., Dotson, N., Bressler, S., Gray, C. (2012). Content-specific fronto-parietal synchronization during visual working memory. Science 338(6110), 1097-1100. https://dx.doi.org/10. $1126 /$ science. 1224000 .

Scemes, E., Giaume, C. (2006). Astrocyte calcium waves: What they are and what they do. Glia 54(7), 716-725. https://dx.doi.org/10.1002/glia.20374.

Schulman, L. (1981). Techniques and Applications of Path Integration. J. Wiley \& Sons, New York.

Schulten, K. (1999). Quantum mechanics. Technical Report Lecture Notes, U. Illinois, Urbana, IL. http://www.ks.uiuc.edu/Services/Class/PHYS480/.

Szu, H., Hartley, R. (1987). Fast simulated annealing. Physics Letters A 122(3-4), 157-162.

vanCreveld, M. (1985). Command in War. Harvard University Press, Cambridge, MA.

vanLaarhoven, P., Aarts, E. (1987). Simulated Annealing: Theory and Applications. D. Reidel, Dordrecht, The Netherlands.

Volterra, A., Liaudet, N., Savtchouk, I. (2014). Astrocyte Ca2+ signalling: an unexpected complexity. Nature Reviews Neuroscience 15, 327-335.

Wehner, M., Wolfer, W. (1983a). Numerical evaluation of path-integral solutions to fokker-planck equations. I. Physical Review A 27, 2663-2670.

Wehner, M., Wolfer, W. (1983b). Numerical evaluation of path-integral solutions to fokker-planck equations. II. restricted stochastic processes. Physical Review A 28, 3003-3011.

Wehner, M., Wolfer, W. (1987). Numerical evaluation of path integral solutions to fokker-planck equations. III. time and functionally dependent coefficients. Physical Review A 35, 1795-1801.

Wu, S., Wang, L., Yi, X. (2012). Time-dependent decoherence-free subspace. Journal of Physics A 405305, 1-11.

Zhang, G., Simon, H. (1985). STM capacity for chinese words and idioms: Chunking and acoustical loop hypotheses. Memory \& Cognition 13, 193-201. 